\title{
Insights into iguanodontian dental architecture from an Early Cretaceous Chinese basal hadrosauriform maxilla (Ornithischia: Iguanodontia)
}

Basal hadrosauriform iguanodontian dinosaurs have been invaluable towards understanding the evolution of the complex and highly efficient advanced hadrosauriform tooth battery dental system. Here we report a new basal hadrosauriform maxilla specimen - IVPP V22529 - from the Dashuiguo Formation of Maortu, Nei Mongol, China that preserves

a corrugated middle ventrolateral margin that differs from the straight and undulating ventral margins found in most iguandontian and non-iguanodontian dinosaurs. The uniqueness of this ventrolateral margin relates to a new dental structure - cementum 'jackets' that wrap about the labial sides of the teeth. To our knowledge this is the first time that cementum has been described migrated onto the tooth crowns of iguandontians (and other dinosaurs), but this trait is common amongst mammals. This dental morphology - seen in a similar form in the basal hadrosauriform Equijubus - therefore broadens our knowledge of iguanodontian maxillary anatomy and shows that the basal hadrosauriform dental system was more morphologically complex than previously thought. IVPP V22529 resembles maxillae specimens of Probactrosaurus gobiensis, a contemporaneous taxon known from the same locality in North China, in sharing an inferred subtriangular shape, a relatively flat lateral surface bearing a low row of foramina as well as similar-looking teeth. However, the presence of a unique corrugated middle ventrolateral margin in IVPP V22529, a low row of foramina on its lateral surface that also open anteriorly and increase in size posteriorly as well as a prominent medial shelf suggests that this specimen does not belong to $P$. gobiensis. However, these differences could conceivably be related to ontogenetic and sexual variation, which have not been fully documented in P. gobiensis. More detailed comparisons of IVPP V22529 and Probactrosaurus are also hampered by the missing posterior portion of IVPP V22529 as well as the missing anterior ramii in 
Probactrosaurus maxillae specimens. It is clear though that IVPP V22529 is different from the more advanced Northern Chinese hadrosauriforms Bactrosaurus and Gilmoreosaurus. The latter lack well-developed maxillary grooves on their medial shelves, unlike IVPP V22529, but all three taxa possess less-developed ones on the medial surfaces of the anteromedial processes of the anterior ramii. Different to IVPP V22529, Gilmoreosaurus also has foramina that are more highly-positioned on the lateral surface of its maxilla as well as a row of larger and more circular 'special' foramina on its medial surface. Thus, at this time, IVPP V22529 is identified as a basal hadrosauriform and not as a new genus or as a new species of Probactrosaurus. 


\section{Insights into iguanodontian dental architecture from an Early Cretaceous Chinese basal hadrosauriform maxilla (Ornithischia: Iguanodontia)}

Michael Pittman, Xing Xu, Jason R Ali, Rui Pei, Waisum Ma, Jin Meng, Shundong Bi

Basal hadrosauriform iguanodontian dinosaurs have been invaluable towards understanding the evolution of the complex and highly efficient advanced hadrosauriform tooth battery dental system. Here we report a new basal hadrosauriform maxilla specimen - IVPP V22529 - from the Dashuiguo Formation of Maortu, Nei Mongol, China that preserves a corrugated middle ventrolateral margin that differs from the straight and undulating ventral margins found in most iguandontian and non-iguanodontian dinosaurs. The uniqueness of this ventrolateral margin relates to a new dental structure - cementum 'jackets' that wrap about the labial sides of the teeth. To our knowledge this is the first time that cementum has been described migrated onto the tooth crowns of iguandontians (and other dinosaurs), but this trait is common amongst mammals. This dental morphology - seen in a similar form in the basal hadrosauriform Equijubus - therefore broadens our knowledge of iguanodontian maxillary anatomy and shows that the basal hadrosauriform dental system was more morphologically complex than previously thought. IVPP V22529 resembles maxillae specimens of Probactrosaurus gobiensis, a contemporaneous taxon known from the same locality in North China, in sharing an inferred subtriangular shape, a relatively flat lateral surface bearing a low row of foramina as well as similar-looking teeth. However, the presence of a unique corrugated middle ventrolateral margin in IVPP V22529, a low row of foramina on its lateral surface that also open anteriorly and increase in size posteriorly as well as a prominent medial shelf suggests that this specimen does not belong to $P$. gobiensis. However, these differences could conceivably be related to ontogenetic and sexual variation, which have not been fully documented in $P$. gobiensis. More detailed comparisons of IVPP V22529 and Probactrosaurus are also hampered by the missing posterior portion of IVPP V22529 as well as the missing anterior ramii in Probactrosaurus maxillae specimens. It is clear though that IVPP V22529 is different from the more advanced Northern Chinese hadrosauriforms Bactrosaurus and Gilmoreosaurus. The latter lack well-developed maxillary grooves on their medial shelves, unlike IVPP V22529, but all three taxa possess less-developed ones on the medial surfaces of the anteromedial processes of the anterior ramii. Different to IVPP V22529, Gilmoreosaurus 
also has foramina that are more highly-positioned on the lateral surface of its maxilla as well as a row of larger and more circular 'special' foramina on its medial surface. Thus, at this time, IVPP V22529 is identified as a basal hadrosauriform and not as a new genus or as a new species of Probactrosaurus. 
NOT PEER-REVIEWED

Insights into iguanodontian dental architecture from an Early Cretaceous Chinese basal hadrosauriform maxilla (Ornithischia: Iguanodontia)

3

4

Michael Pittman ${ }^{1}$, Xing Xu ${ }^{2}$, Jason R. $\mathrm{Ali}^{1}$, Rui Pei ${ }^{1,4}$, Waisum $\mathrm{Ma}^{1}$, Jin Meng ${ }^{2,3}$ \& Shundong $\mathrm{Bi}^{2,4}$

${ }^{1}$ Vertebrate Palaeontology Laboratory, Life and Planetary Evolution Research Group, Department of Earth Sciences, The University of Hong Kong, Pokfulam, Hong Kong.

${ }^{2}$ Key Laboratory of Vertebrate Evolution and Human Origins, Institute of Vertebrate Paleontology \& Paleoanthropology, Chinese Academy of Sciences, 142 Xizhimenwai Street, Beijing, 100044, China.

${ }^{3}$ Department of Biology, Indiana University of Pennsylvania, Indiana, Pennsylvania 15705, USA.

${ }^{4}$ Division of Paleontology, American Museum of Natural History, Central Park West and 79th Street, New York, New York 10024, USA.

*Corresponding author: mpittman@hku.hk

Keywords: basal hadrosauriform, iguanodontian, Probactrosaurus, dental architecture, tooth root, tooth socket, cementum, periodontal ligament, maxillary teeth, maxilla, dinosaur, Maortu, Nei Mongol, Early Cretaceous

\begin{abstract}
Basal hadrosauriform iguanodontian dinosaurs have been invaluable towards understanding the evolution of the complex and highly efficient advanced hadrosauriform tooth battery dental system. Here we report a new basal hadrosauriform maxilla specimen - IVPP V22529 - from the Dashuiguo Formation of Maortu, Nei Mongol, China that preserves a corrugated middle ventrolateral margin that differs from the straight and undulating ventral margins found in most iguandontian and non-iguanodontian dinosaurs. The uniqueness of this ventrolateral margin relates to a new dental structure - cementum 'jackets' that wrap about the labial sides of the teeth. To our knowledge this is the first time that cementum has been described migrated onto the tooth crowns of iguandontians (and other dinosaurs), but this trait is common amongst mammals. This dental morphology - seen in a similar form in the basal hadrosauriform Equijubus - therefore broadens our knowledge of iguanodontian maxillary anatomy and shows that the basal hadrosauriform dental system was more morphologically complex than previously thought. IVPP V22529 resembles maxillae specimens of Probactrosaurus gobiensis, a contemporaneous taxon known from the same locality in North China, in sharing an inferred subtriangular shape, a relatively flat lateral surface bearing a low row of foramina as well as similar-looking teeth. However, the presence of a unique corrugated middle ventrolateral margin in IVPP V22529, a low row of foramina on its lateral surface that also open anteriorly and increase in size posteriorly as well as a prominent medial shelf suggests that this specimen does not belong to $P$. gobiensis. However, these differences could conceivably be related to ontogenetic and sexual variation, which have not been fully documented in P. gobiensis. More detailed comparisons of IVPP V22529 and
\end{abstract}


Probactrosaurus are also hampered by the missing posterior portion of IVPP V22529 as well as the missing anterior ramii in Probactrosaurus maxillae specimens. It is clear though that IVPP V22529 is different from the more advanced Northern Chinese hadrosauriforms Bactrosaurus and Gilmoreosaurus. The latter lack well-developed maxillary grooves on their medial shelves, unlike IVPP V22529, but all three taxa possess less-developed ones on the medial surfaces of the anteromedial processes of the anterior ramii. Different to IVPP V22529, Gilmoreosaurus also has foramina that are more highly-positioned on the lateral surface of its maxilla as well as a row of larger and more circular 'special' foramina on its medial surface. Thus, at this time, IVPP V22529 is identified as a basal hadrosauriform and not as a new genus or as a new species of Probactrosaurus.

\section{Introduction}

Maortu (Chow \& Rozhdestvensky, 1960: = Maorty; 毛尔图) is a fossil locality of Early Cretaceous age (Dashuiguo Formation: Barremian to Albian stages (Rozhdestvensky, 1966; Rozhdestvensky, 1974; van Itterbeeck et al., 2001; van Itterbeeck et al., 2004) located approximately half-way along China's northern frontier in Nei Mongol Autonomous Region ( 内蒙古自治区), 500km west of the provincial capital Hohhot (呼和浩特市) (Fig. 1).

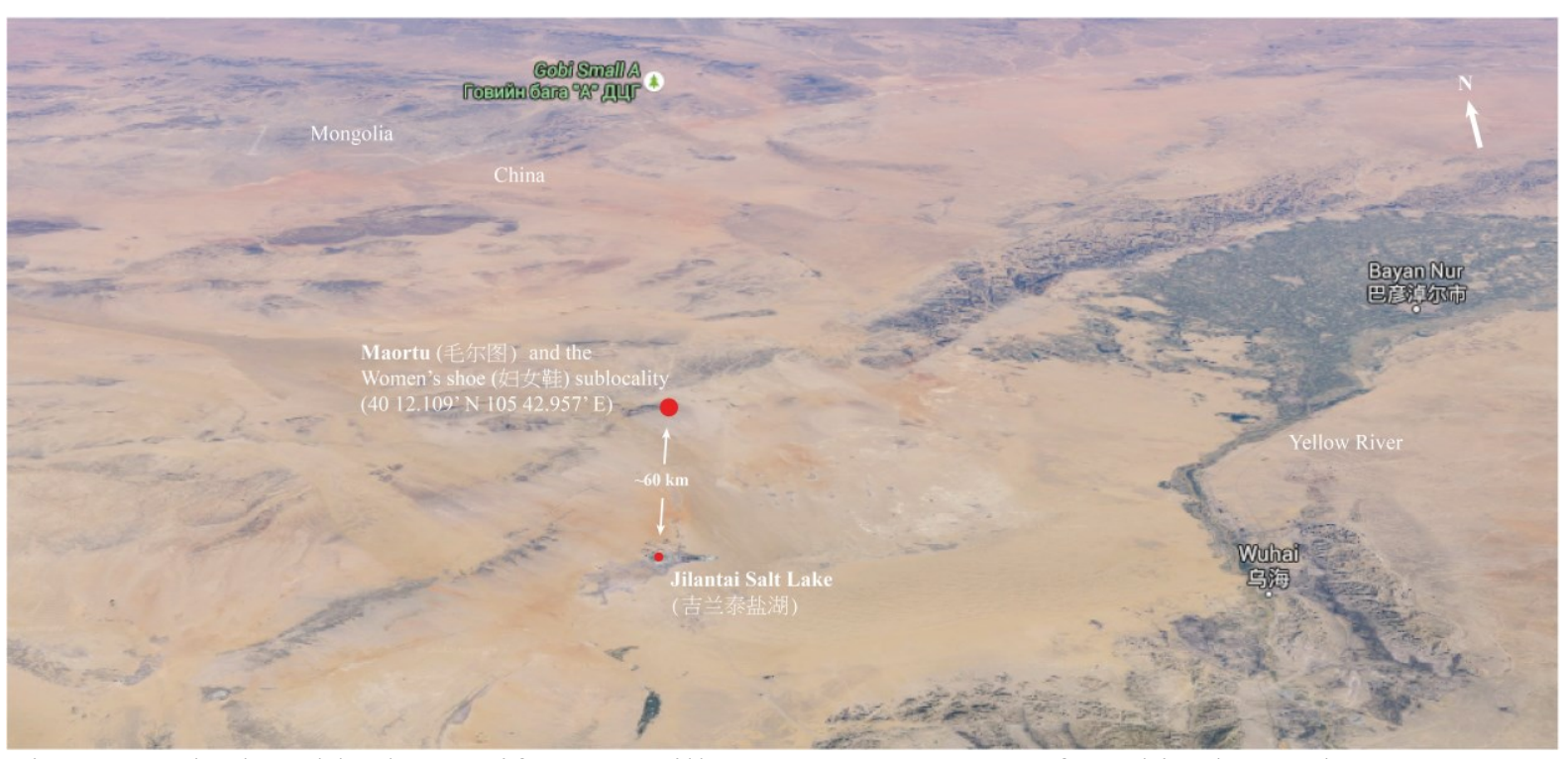

Figure 1. The basal hadrosauriform maxilla IVPP V22529 was found in the Early Cretaceous Dashuiguo Formation of Maortu, Nei Mongol, China (map produced from a Google Maps image). Maortu is the type locality of the non-euhadrosaurian hadrosauriform Probactrosaurus gobiensis (Rozhdestvensky, 1966).

Maortu is the type locality of three dinosaurs: the non-euhadrosaurian hadrosauriform Probactrosaurus gobiensis (Rozhdestvensky, 1966) [this study follows the ornithopod classification of Norman (2015)], the advanced non-carcharodontosaurine carcharodontosaurid Shaochilong maortuensis (Brusatte et al., 2009; Brusatte et al., 2010) and the basal non-ankylosaurine ankylosaurid Gobisaurus domoculus (Vickaryous et al., 2001). It is also the type locality of the trionychine trionychid turtle Dongania maortuensis (Hans-Volker, 1999; Yeh, 1965 [See Vitek \& Danilov, 2010 for taxonomic discussion]). In the summer of 2014 a team including several of the authors (MP, JRA, JM and SDB) visited Maortu (and its surrounding areas) where they recovered a variety of fragmentary and mostly isolated dinosaur and mammal bones now housed at the Institute of Vertebrate Paleontology 
and Paleoanthropology (IVPP), Beijing. Amongst the largest of these bones is an isolated, crushed and posteriorly broken right iguanodontian maxilla $(\sim 12 \mathrm{~cm}$ tall and $22 \mathrm{~cm}$ long; Fig. $3)$.

Early Cretaceous Asian iguanodontian maxillae are known from nine Chinese taxa (Bactrosaurus johnsoni [Prieto-Márquez, 2011], Bolong yixianensis [Wu et al., 2010; Zheng et al., 2013], Equijubus normani [You et al., 2003c], Jinzhousaurus yangi [Wang \& Xu, 2001], Lanzhousaurus magnidens [You et al., 2005], Probactrosaurus gobiensis [Norman, 2002; Rozhdestvensky, 1966], P. mazongshanensis [Lü, 1997; Norman, 2002], Shuangmiaosaurus gilmorei [You et al., 2003a] and Xuwulong yueluni [You et al., 2011]), two Japanese taxa (Fukuisaurus tetoriensis [Kobayashi \& Azuma, 2003; Shibata \& Azuma, 2015] and Koshisaurus katsuyama [Shibata \& Azuma, 2015]), two Kazak taxa (Altirhinus kurzanovi [Norman, 1998] and Batyrosaurus rozhdestvenskyi [Godefroit et al., 2012]) and a Thai specimen identified to a higher taxonomic level (Siamodon nimngami; Buffetaut \& Suteethorn, 2011; nomen dubium: Norman, 2015). Comparisons between IVPP V22529 and the aforementioned taxa (Table 1) identifies IVPP V22529 as a non-euhadrosaurian hadrosauriform based on the presence of at least two replacement maxillary crowns and the absence of a single median primary ridge on the teeth (see Description and Comparison and Discussion). The presence of marginal denticles comprising of parallel ledges with single rows of $\sim 6$ relatively large mammillae suggests that IVPP V22529 is a basal hadrosauriform.

\begin{tabular}{|c|c|c|c|}
\hline Taxon & Specimen number & Material & References \\
\hline \multicolumn{4}{|l|}{ China } \\
\hline IVPP V22529 & $\begin{array}{l}\text { IVPP V22529 (field } \\
\text { number: JLT } \\
\text { 20140622-1) }\end{array}$ & $\begin{array}{l}\text { Partial right maxilla } \\
\text { (posterior ramus } \\
\text { missing) }\end{array}$ & $\begin{array}{l}\text { This study (Pittman } \\
\text { et al. 2015) }\end{array}$ \\
\hline \multirow[t]{4}{*}{$\begin{array}{l}\text { Bactrosaurus } \\
\text { johnsoni*^}\end{array}$} & $\begin{array}{l}\text { AMNH } 6553 \\
\text { (holotype) }\end{array}$ & $\begin{array}{l}\text { Adult } / \text { subadult left } \\
\text { maxilla }\end{array}$ & $\begin{array}{l}\text { Prieto-Márquez, } \\
\text { 2011: Figs. 7, } 8\end{array}$ \\
\hline & $\begin{array}{l}\text { AMNH 6390-6393, } \\
6514\end{array}$ & $\begin{array}{l}\text { Juvenile left maxillae } \\
\text { (AMNH } 6393 \text { is a } \\
\text { partial specimen) }\end{array}$ & $\begin{array}{l}\text { Prieto-Márquez, } \\
\text { 2011: Figs. 11, } 12\end{array}$ \\
\hline & $\begin{array}{l}\text { AMNH 6388, 6389, } \\
6583\end{array}$ & $\begin{array}{l}\text { Juvenile right } \\
\text { maxillae }\end{array}$ & $\begin{array}{l}\text { Prieto-Márquez, } \\
\text { 2011: Figs. 9, } 10\end{array}$ \\
\hline & 3 SBDE 1 & $?$ & Godefroit et al., 1998 \\
\hline \multirow[t]{2}{*}{ Bolong yixianensis* } & YHZ-001 & Left maxilla & $\begin{array}{l}\text { Wu et al., } 2010 \\
\text { Wu \& Godefroit, } \\
\text { 2012: Figs. } 19.2 \text {, } \\
\text { 19.3 }\end{array}$ \\
\hline & ZMNH-M8812 & $\begin{array}{l}\text { Juvenile left and } \\
\text { right maxillae }\end{array}$ & $\begin{array}{l}\text { Zheng et al., 2013: } \\
\text { Figs. 2, 4-6 }\end{array}$ \\
\hline Equijubus normani & $\begin{array}{l}\text { IVPP V12534 } \\
\text { (holotype) }\end{array}$ & $\begin{array}{l}\text { Complete articulated } \\
\text { maxilla exposed on } \\
\text { both the right and left } \\
\text { lateral sides. }\end{array}$ & $\begin{array}{l}\text { You et al., 2003c: } \\
\text { Fig. } 1\end{array}$ \\
\hline Jinzhousaurus yangi & $\begin{array}{l}\text { IVPP V12691 } \\
\text { (holotype) }\end{array}$ & $\begin{array}{l}\text { Complete articulated } \\
\text { maxilla exposed on } \\
\text { its left lateral side; } \\
\text { medial side } \\
\text { embedded in matrix. }\end{array}$ & $\begin{array}{l}\text { Wang \& Xu, 2001: } \\
\text { Figs. 1, 2; Barrett et } \\
\text { al., 2009: Fig. } 1\end{array}$ \\
\hline Lanzhousaurus & GSLTZP01-001 & Isolated maxillary & You et al., 2005: Fig. \\
\hline
\end{tabular}


magnidens*

Probactrosaurus

gobiensis

(holotype)

PIN 2232/9-2*

PIN 2232/10-2*

P. mazongshanensis

IVPP V1134.10-15

**

Shuangmiaosaurus

gilmore $^{* \#}$

Xuwulong yueluni*

LPM 0165

GSGM-F00001

Kazakhstan

Altirhinus kurzanovi*

Batyrosaurus

rozhdestvenskyi*

PIN 3386/7

AEHM 4/1

Japan

Fukuisaurus

tetoriensis*

FPDM-V-40-1
(holotype)
FPDM-V-40-5
FPDM-V-40-13

Koshisaurus

katsuyama*

FPDM-V9079

Thailand

Siamodon

nimngami* (nomen

PRC-4

dubium: Norman,

2015

\section{Materials and Methods}

teeth

Partial right maxilla

(missing anterior

ramus)

Partial right maxilla

(missing anterior

ramus as well as

teeth from anterior

and posterior aveolar

slots)

Isolated maxillary

teeth

Left maxilla

Both maxillae

Both maxillae

$\sim 30$ maxillary teeth

Right maxilla

Left maxilla

Isolated left maxillary tooth

Right maxilla

\section{A-H}

Norman, 2002: Fig. 5; Rozhdestvensky, 1966

Norman, 2002: Fig. 5; Rozhdestvensky, 1966

Lü, 1997; Norman, 2002: Fig. 4A

You et al., 2003a:

Fig. 1

You et al., 2011:

Figs. 2, 3

Norman, 1998: Fig. 6

Godefroit et al., 2012: Fig. 20.10C, D

Kobayashi \& Azuma, 2003: Fig. 6

Kobayashi \& Azuma, 2003: Fig. 2C-E

Kobayashi \& Azuma, 2003

Shibata \& Azuma, 2015: Fig. 3

Left maxilla

Buffetaut \&

Suteethorn, 2011:

Fig. 1; Norman, 2015

(nomen dubium)

*Taxa studied from the literature only; **Specimens absent from host collection; ^Upper Cretaceous taxa that were also found in Nei Mongol; " Suggested to be an Upper Cretaceous taxon by You et al. (2003a) based on biostratigraphic evidence.

Table 1. Early Cretaceous Asian iguanodontian dinosaur maxillae studied.

List of Early Cretaceous Asian iguanodontian maxillae used to describe IVPP V22529.

IVPP V22529, an isolated partial right iguanodontian maxilla. This specimen was excavated, studied and described using standard palaeontological methods, in accordance with a fossil excavation permit (14-0620-JLT) obtained from the Department of Land and Resources, Nei Mongol, China. 


\section{Locality and Horizon}

Maortu (毛尔图), “Women’s shoe” (妇女鞋) sublocality (400 12.109’ N 105 42.957’ E); $\sim 60 \mathrm{~km}$ north of Jilantai lake (吉兰泰盐湖), Alxa Left Banner, Alashan League, Nei Mongol, China (Figs. 1, 2); Dashuiguo Formation, Barremian to Albian, Early Cretaceous (Rozhdestvensky, 1966; Rozhdestvensky, 1974; van Itterbeeck et al., 2001; van Itterbeeck et al., 2004).

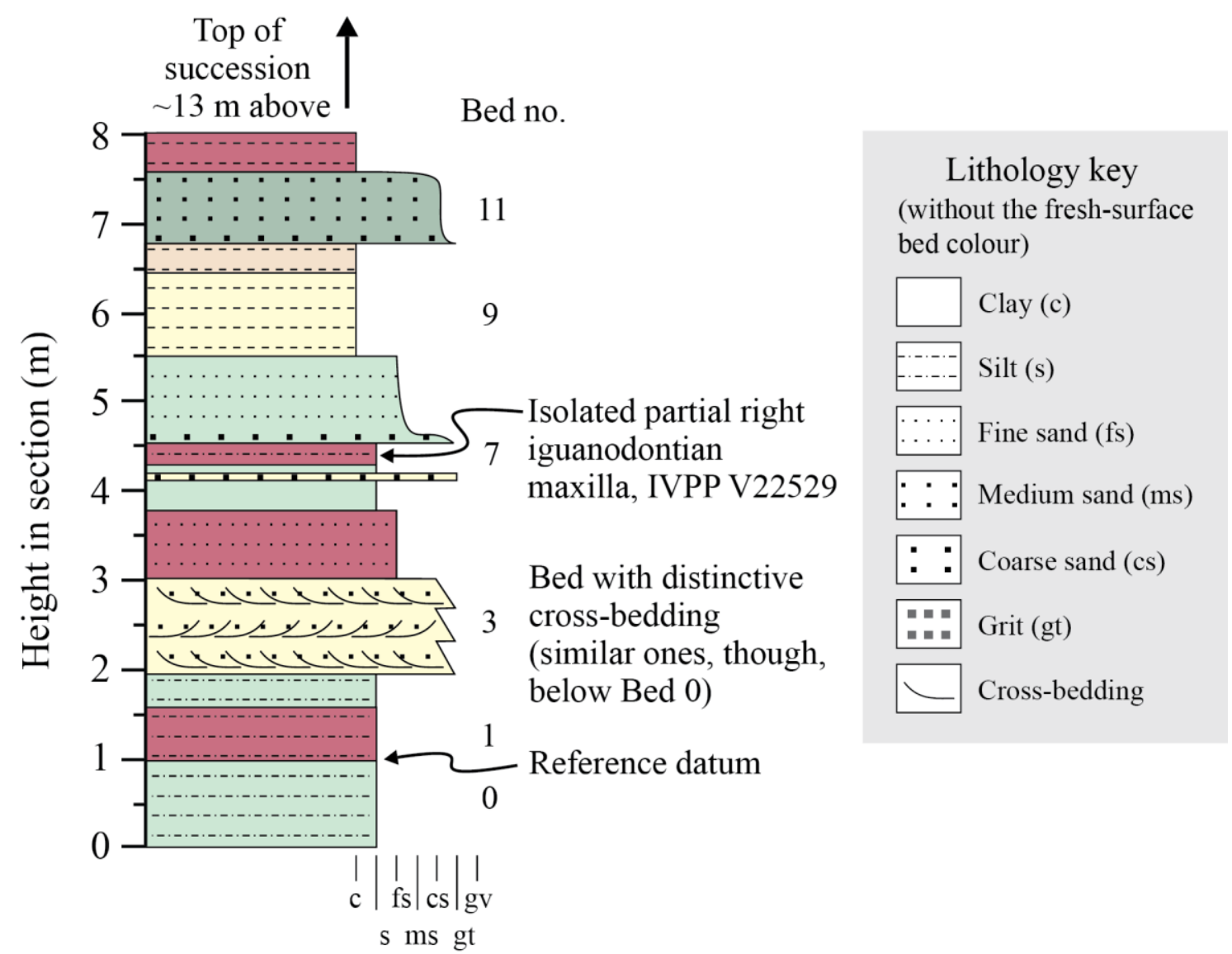

Figure 2. Stratigraphic log showing the position of IVPP V22529 in the local rock succession at the “Women's shoe” (妇女鞋) sublocality (40⒓109' N 105 42.957' E). Grain size abbreviations: c, clay; s, silt; fs, fine sand; ms, medium sand; cs, coarse sand; gt, grit; gv, gravel.

IVPP V22529 was recovered from the lower part of the exposure at the "Women's shoe" sublocality (Bed 7 in Fig. 2) (40 12.109' N $105^{\circ} 42.957^{\prime}$ E) located $\sim 60 \mathrm{~km}$ north of Jilantai lake in the Alxa Left Banner of the Alashan League of Nei Mongol, China. The specimen was found $\sim 13$ metres below where the Early Cretaceous succession is capped by recent gravel washout material. The sediments comprise vari-coloured beds (red-purple, pale green, olive green and cream) that are typically $20-100 \mathrm{~cm}$ thick. The grain size is mostly clay or silt, but coarse sands and grits are encountered. Some units show cross-bedding (Bed 3 in Fig. 2), but most are devoid of any internal layering, apart from a few that fine upwards. The depositional setting is inferred to be a lake margin, the cross-bedded horizons probably marking a time when a stream or small river, quite probably ephemeral, was discharging into the system. 
147

148

149

150

151

152

153

154

\section{Description and Comparison}

IVPP V22529 is an isolated right iguanodontian maxilla that is missing its posterior ramus and has a broken anterior one (Fig. 3). This section first describes the specimen's dentition and compares it with other iguanodontians because this portion of the maxilla contains the most diagnostic and unique information in this specimen. Then, the maxillary body will be described and compared with other iguanodontians.

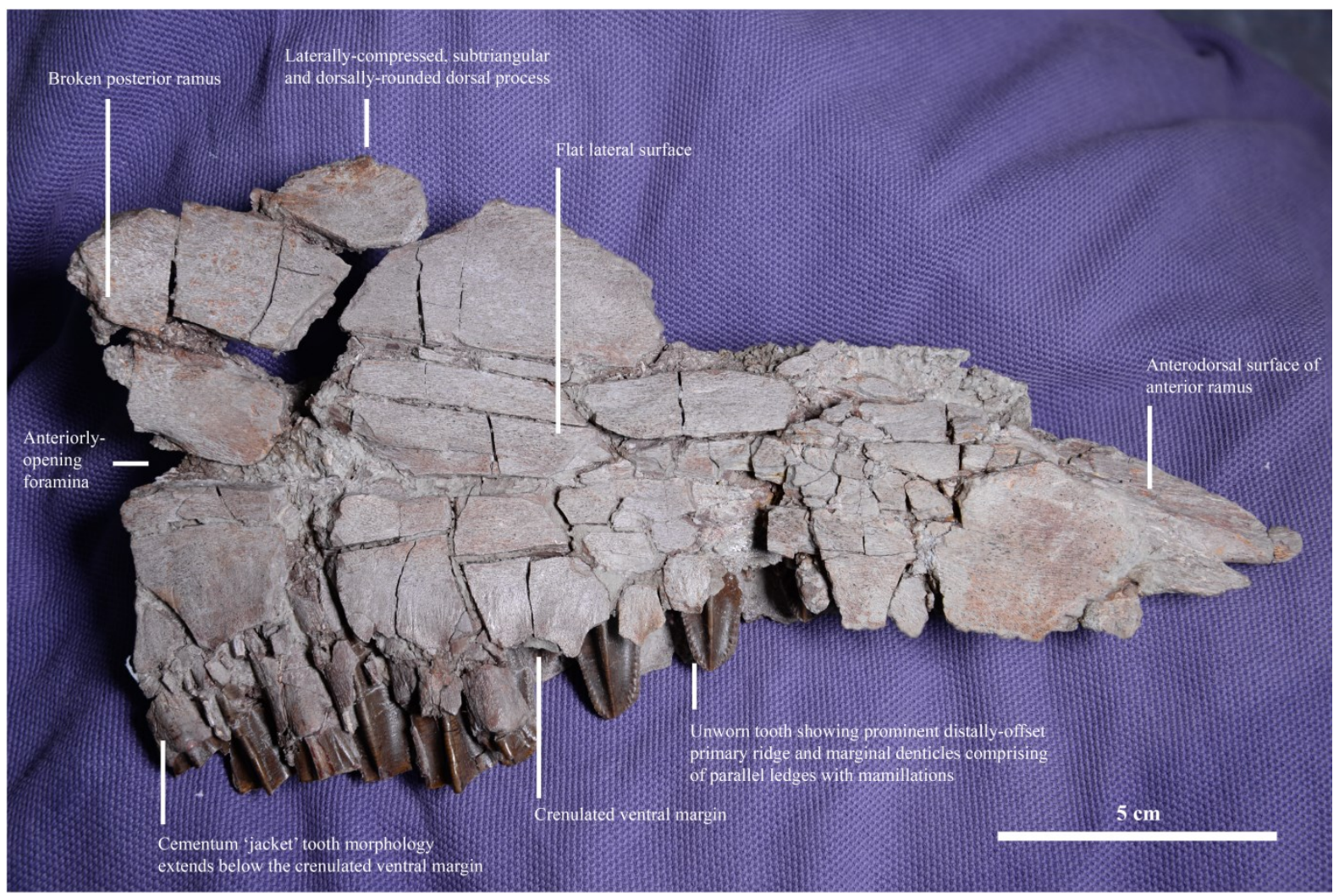

Figure 3. IVPP V22529 in lateral view showing the missing posterior ramus and broken anterior one, the dorsal process and lateral surface, the labial view of the tooth row and the corrugated middle ventrolateral surface formed by an unusual cementum 'jacket' tooth morphology. 


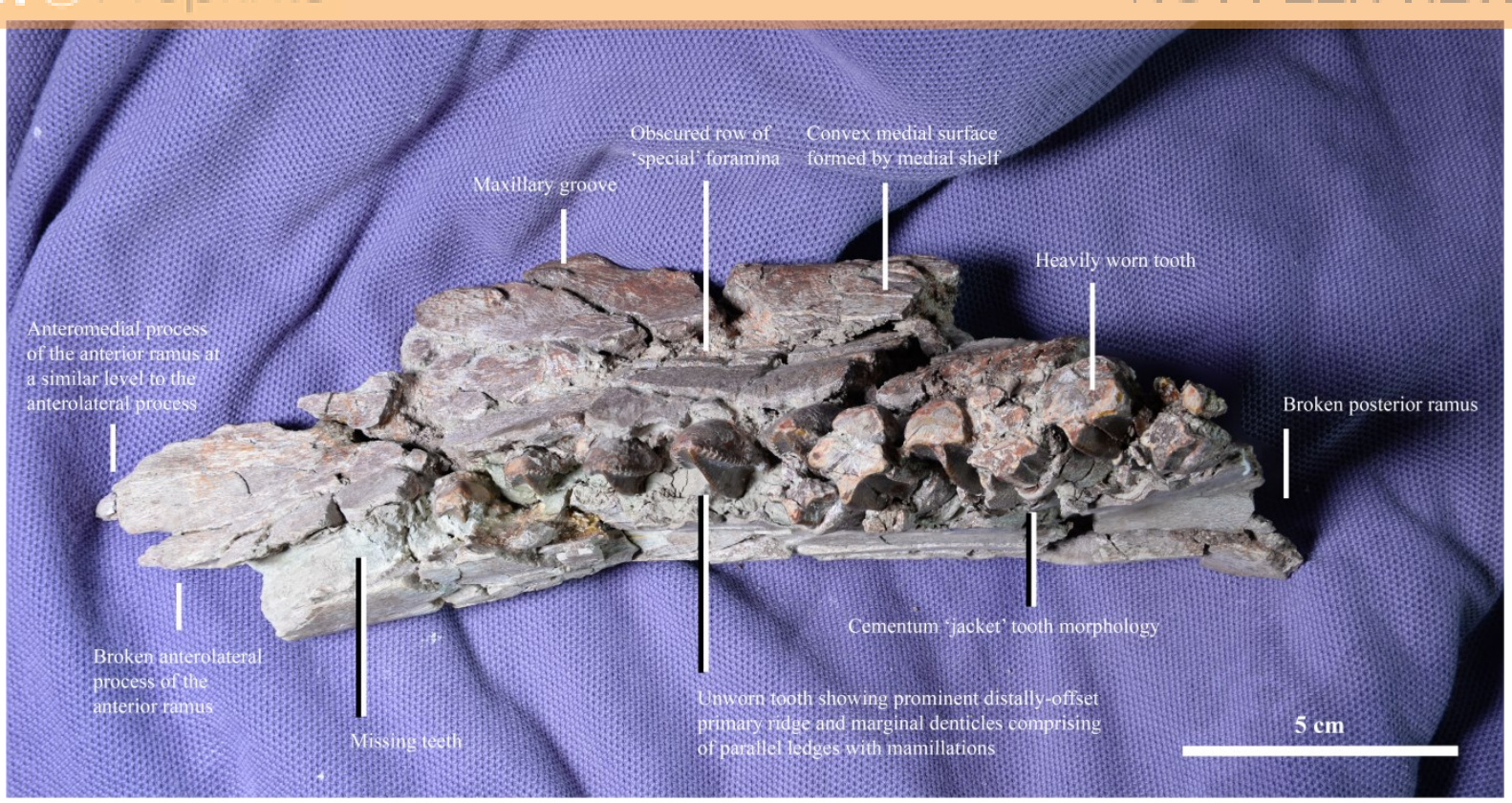

Figure 4. Tooth row in ventral view showing the specimen's partial and heavily worn teeth, its unworn teeth as well as its missing ones.

IVPP V22529 preserves a partial tooth row (Figs. 2, 3) comprising of more than 18 vertical tooth positions, as indicated by 14 in situ teeth, an empty aveolar socket and a row of at least three empty parallel aveolar sockets at the anterior end of the maxilla (although the empty sockets may each have accommodated more than one tooth (Norman, 2002)). Without the posterior portion of the maxillary tooth row and no associated complete dentary row to estimate the number of vertical maxillary tooth positions, the latter is uncertain.

Nevertheless, by comparison, the non-euhadrosaurian hadrosauriform Probactrosaurus (PIN 2232/9-2, /10-2; Norman, 2002) preserves $\sim 17$ vertical maxillary tooth positions out of an estimated total of 22+ positions (22-23 positions estimated by Norman (2002); 23 or more total positions estimated by Rozhdestvensky (1966)). The 'iguanodontoid' styracosternan Jinzhousaurus is estimated to have fewer maxillary teeth than Probactrosaurus with only 15-16 teeth [IVPP V12691, Barrett et al., 2009: Fig. 3C]), as in the hadrosauriform Koshisaurus which has 19 vertical tooth positions [FPDM-V907, Shibata \& Azuma, 2015: Figs. 3, 8]. The basal non-euhadrosaurian hadrosauriform Altirhinus may have had a slightly higher vertical tooth position count than Probactrosaurus as Norman (1998) estimated 26 positions on the basis of the 24 positions present in its dentary (PIN 3386/7, Norman, 1998: Fig. 16). However, Altirhinus only preserves direct evidence of 21 vertical maxillary tooth positions (right maxilla of PIN 3386/7; Norman, 1998: Fig. 6). Given the uncertain tooth position count in IVPP V22529 it might even be possible that it has a high tooth position count as in the basal non-euhadrosaurian hadrosauriform Eolambia (32 positions in the left maxilla of CEUM 9758; Kirkland, 1998: Fig. 4A-C). Amongst iguanodontians the number of maxillary teeth appears to increase during ontogeny (Horner et al., 2004; Hübner \& Rauhut, 2010; Zheng et al., 2013), but the relatively large size of the maxilla fragment suggests that IVPP V22529 probably has close to its maximum number of vertical tooth positions.

Replacement crowns 


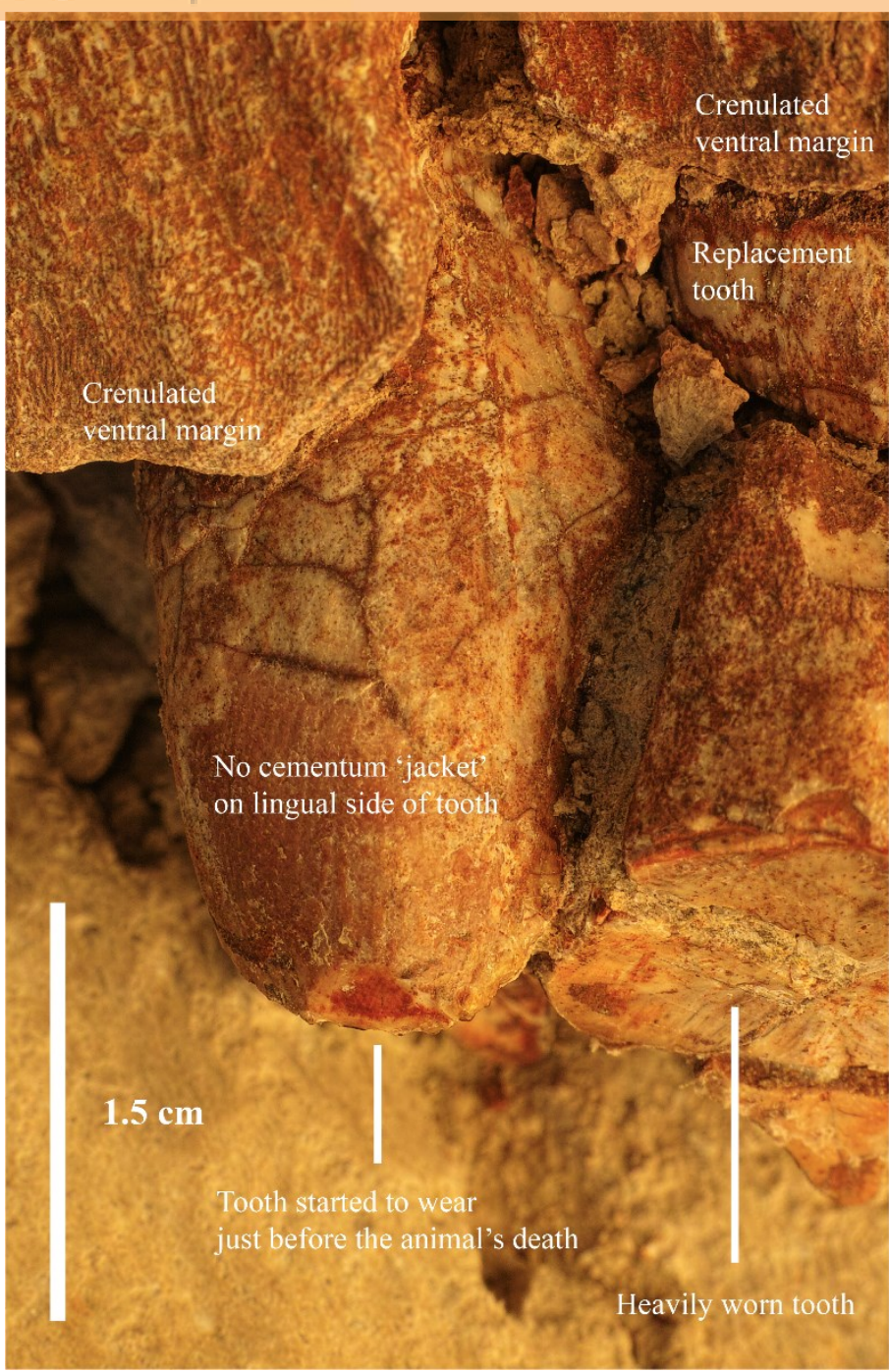

Figure 5. IVPP V22529 appears to be a hadrosauriform iguandontian because it has at least two replacement teeth (Norman, 2015). This is indicated by a heavily worn tooth (right) that is supported by a replacement tooth, which is adjacent to a tooth that had just started to wear before the animal died.

One replacement crown is observed in five positions along the tooth row (Fig. 4), but the preservation of the socket walls prevents the total number of replacement crowns from being determined e.g. two replacement crowns are revealed by the broken posterior portion of the right medial wall of Altirhinus PIN 3386/7 (Norman, 1998: Fig. 6). However, there appears to be indirect evidence of at least two replacement teeth in IVPP V22529 because one heavily worn tooth is supported by a replacement tooth and anterior to it there is a taller erupted tooth that had only just started to be worn prior to the animal's death (Fig. 5). The presence of at least two replacement crowns implies that IVPP V22529 is a hadrosauriform iguandontian (Norman, 2015: character 54, state 1) - non-hadrosauriform iguanodontians have one functional crown supported by only one replacement crown (Norman, 2015: character 54, state 0$)$ ). 


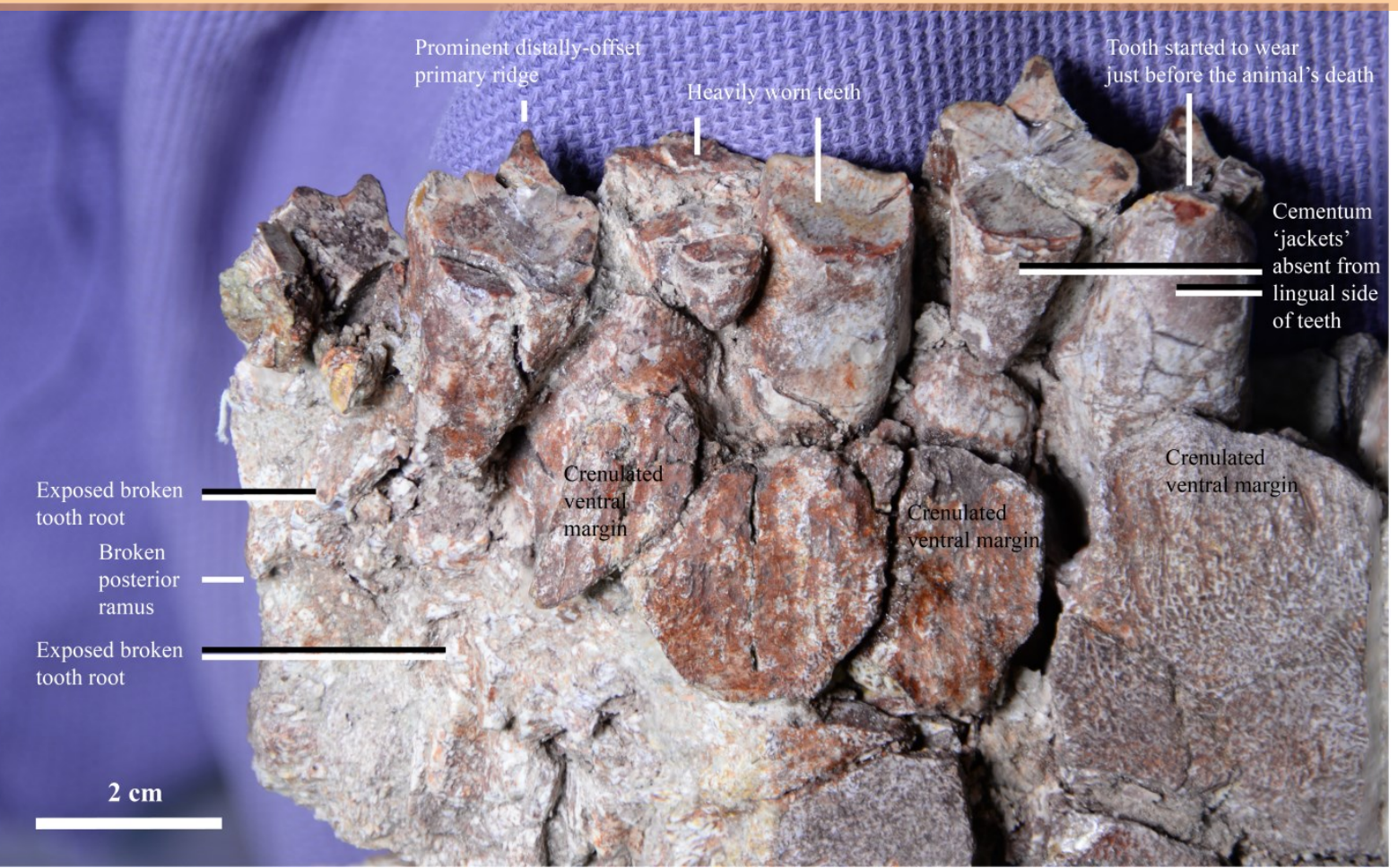

Figure 6. Six heavily worn teeth in IVPP V22529 shown in ventromedial view.

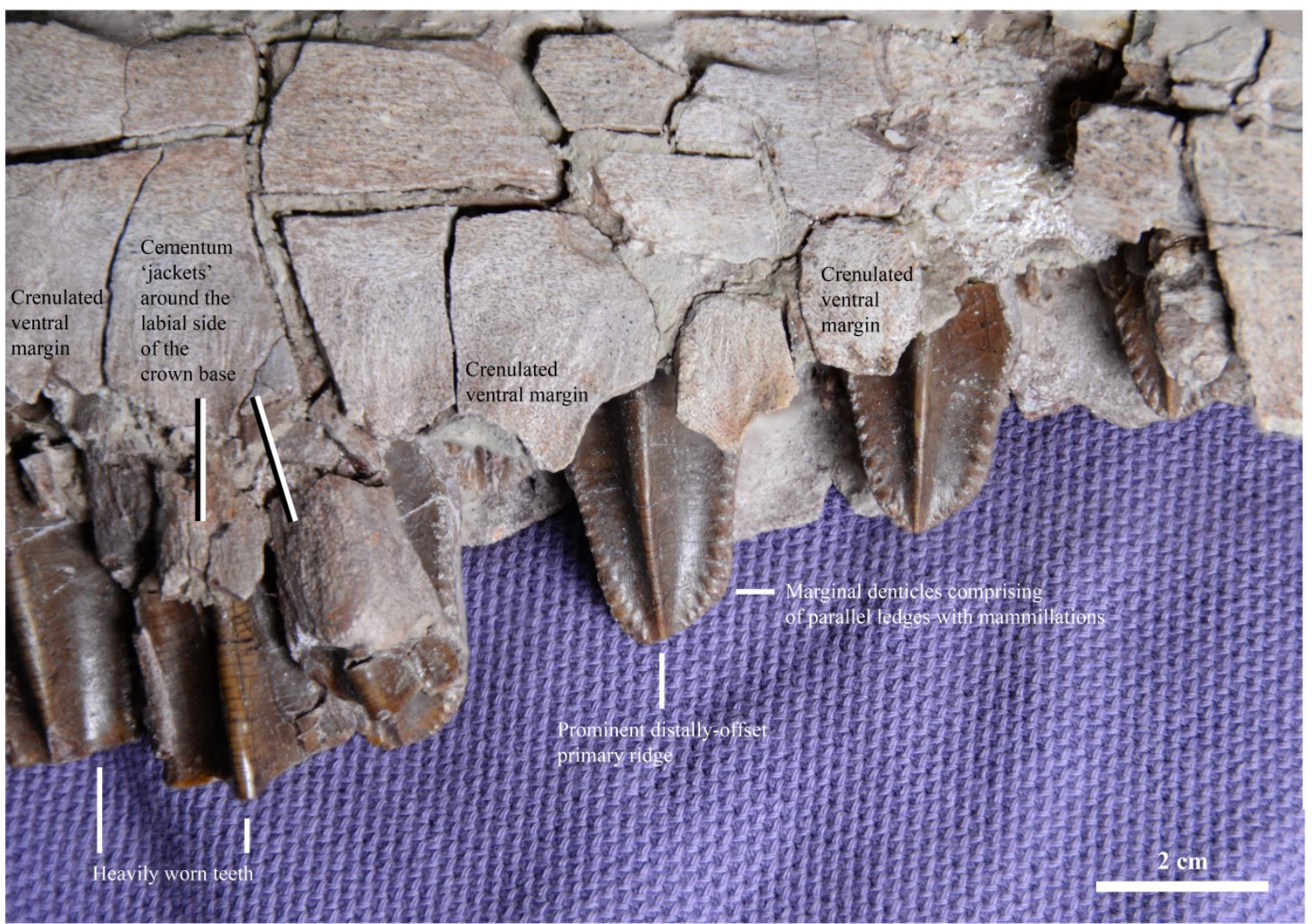

Figure 7. The teeth of IVPP V22529 are dominated by a well-developed distally-offset primary ridge and lack any subsidiary (accessory) ridges. The unworn teeth of IVPP V22529 show marginal denticles comprising of parallel ledges with single rows of $\sim 6$ relatively large mammillae. The latter suggests that IVPP V22529 is a basal hadrosauriform. 
Six of the teeth preserved in IVPP V22529 have well-developed wear facets (Fig. 6) that are up to $2 \mathrm{~cm}$ shorter vertically than the tallest of the three teeth with slightly worn tips (the latter are presumed to have erupted not long before the animal's death). There is a small fragment of a seventh worn tooth located in the most posteriorly preserved position along the tooth row (Fig. 6). One of the extensively worn teeth as well as all of the slightly worn or non-worn teeth have marginal denticles comprising of parallel ledges with single rows of $\sim 6$ mammillae (Fig. 7). Styracosternan iguanodontians have marginal denticles on both their maxillary and dentary teeth that form ledges with mammillations (Norman, 2015: character 58, state 2), but in IVPP V22529 the mammillae are comparatively large suggesting that it is a basal hadrosauriform as more advanced hadrosauriforms have smaller mammillae.

Primary and subsidiary (accessory) ridges

In labial view, the enamelled surface of the crown is narrow and appears lozenge-like (elongated and asymmetrically diamond-shaped), as in Probactrosaurus (Norman, 2002), Altirhinus (Norman, 1998) and Iguanodon (Norman et al., 1987) [Fig. 7]. The crown's asymmetry is indicated by the distal offset of an enlarged primary ridge relative to the tooth's mid-line (a feature diagnostic of Iguanodontia (Norman, 2015: character 68, state 1) which includes Probactrosaurus, Altirhinus and Jinzhousaurus (Norman, 1998; Norman, 2002; Wang \& Xu, 2001)) and the anterior position of the shoulder of the crown margin (as in Altirhinus (PIN 3386/7, Norman, 1998: Fig. 21B) and Jinzhousaurus (IVPP V12691, Wang $\& \mathrm{Xu}, 2001$ ) [Fig. 7]. The absence of a single median primary ridge indicates that IVPP V22529 is not a euhadrosaurian iguanodontian (Norman, 2015: character 68, state 3). As in Probactrosaurus, there appears to be little evidence of subsidiary ridges (Norman, 2002) [Fig. 7], unlike in Altirhinus (Norman, 1998: Fig. 21B) and Jinzhousaurus (IVPP V12691, Wang \& Xu, 2001) where there is one anterior to the primary ridge and unlike in the styracosternan iguanodontian Lanzhousaurus (GSLTZP01-001; You et al., 2005: Fig. 2A) where the primary ridge is flanked by several subsidiary ones. There is no lingual ridge on the maxillary tooth crowns of IVPP V22529, so this ridge still appears to be an autapomorphy of Koshisaurus (FPDM-V907, Shibata \& Azuma, 2015: Figs. 3C, 8B, 9B]). It is worth mentioning that the two isolated near-complete maxillary teeth (IVPP V1134.10) of Probactrosaurus mazongshanensis Lü, 1997 - not a junior synonym of Probactrosaurus gobiensis (Norman, 2002) - could not be located at the IVPP in May 2015 for direct comparison with IVPP V22529. However, Lü (1997) noted that these teeth have a large highly-developed primary ridge and his figure of one of them (his Figure 4) shows very similar morphological traits to the maxillary teeth of IVPP V22529.

Tooth root morphology

Relatively straight but poorly preserved tooth roots are exposed on the broken posteromedial surface of IVPP V22529. One portion of an exposed tooth root appears to be longitudinally grooved (a synapomorphy of Iguanodontia (Norman, 2015: character 59, state 1 DELTRAN)), but this observation is equivocal owing to the root's poor preservation. Hadrosauromorpha is characterised by highly angular-sided roots (hexagonally prismatic) that relate to close packing of the teeth in a functionally integrated multi-tooth magazine (Norman, 2015: character 59, state 2). Given the poor state of tooth root preservation in IVPP V22529, the presence of the latter in the specimen cannot be excluded at present. 


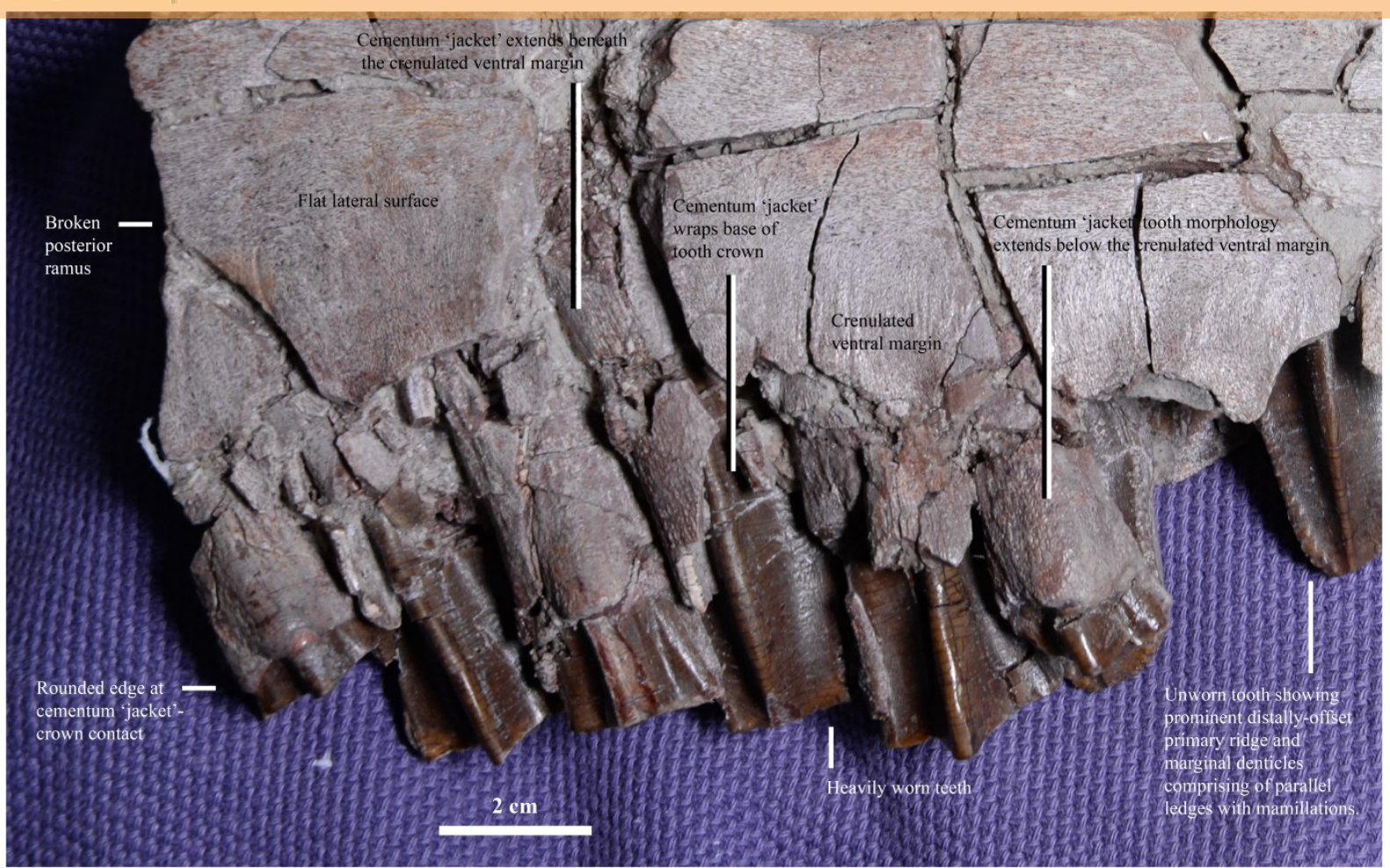

Figure 8. In labial view, the five middle maxillary crowns preserve a bone-like sheath over their base. This appears to be cementum owing to its rugose texture and the absence of the fibres expected in ossified periodontal ligaments. This cementum 'jacket' morphology which is the first to be described amongst dinosaurs to our knowledge - originates within the tooth socket and extends below the crenulated ventral margin of the maxilla to form a corrugated ventrolateral surface created by the grooves that separate each 'jacket'.

Below the five middle maxillary crowns at the broken posterior end of the specimen, the labial side of each tooth appears to be tightly enveloped by smooth to a slightly rugose material (Fig. 8). These bone-like sheaths do not form a continuous surface and appear separate from the walls of the tooth socket. They extend beyond the crenulated ventral margin of the lateral surface, but begin beneath the socket walls themselves as revealed through a broken portion of the wall (Fig. 8). The separation of this structure from the tooth socket walls and its non-uniform roughened texture suggests that it is cementum (Fig. 8). Cementum with similar textural characteristics has been identified in Probactrosaurus (Norman, 2002) and other iguanodontians; this texture is presumably associated with ligamentous scarring on the tooth root. However, the unknown structure could conceivably be ossified periodontal ligaments as these bind the tooth root to its socket. However, this hypothesis is poorly supported as no structures resembling ligamental fibres were observed in IVPP V22529. If these fibres were observed they should also show differences in orientation along the length of the ligament. The lack of pathologies on the 'jackets' suggests that tooth eruption was probably relatively smooth and unhindered. 


\section{Peer Preprints NOT PEER-REVIEWED}

294

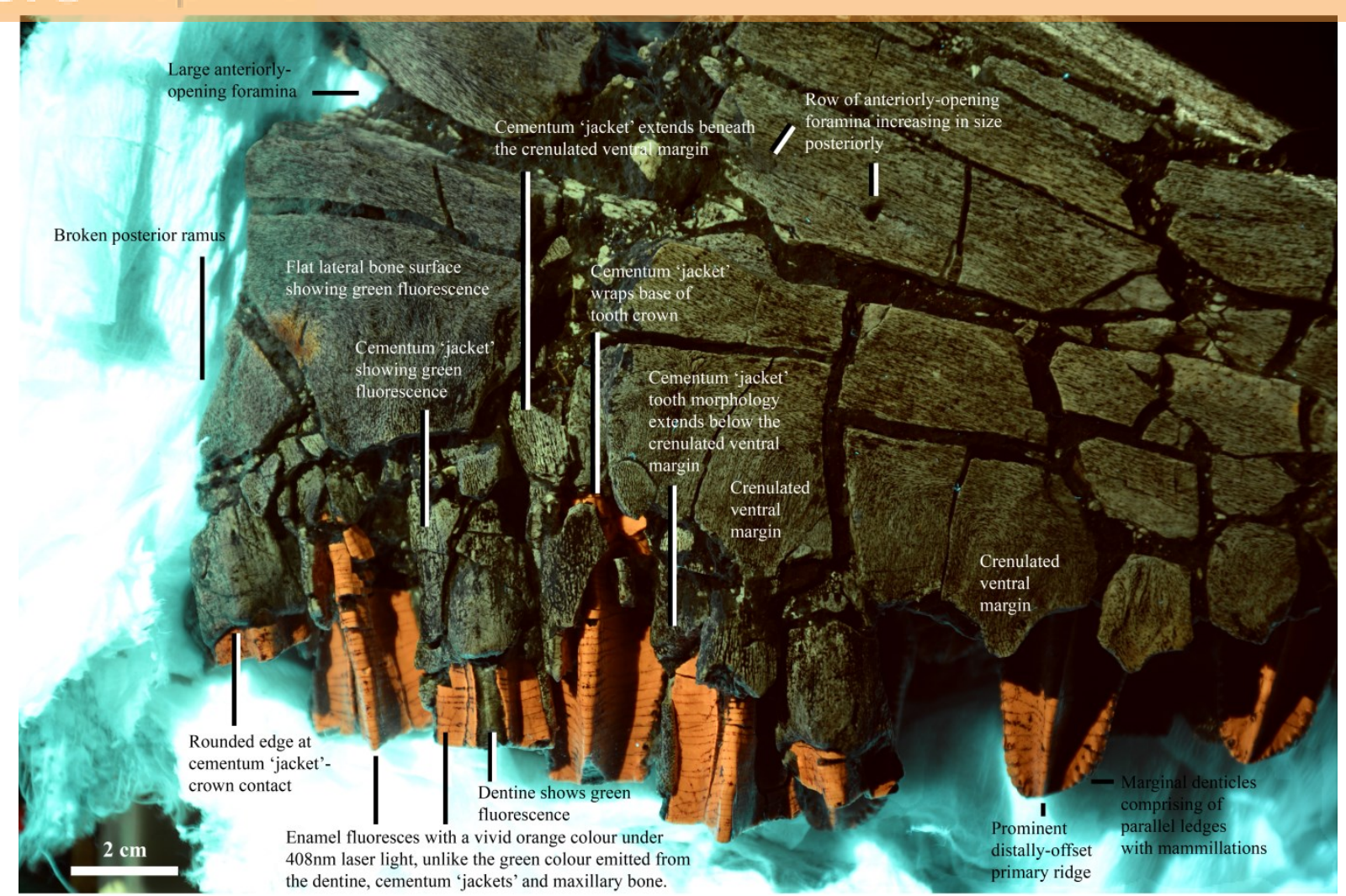

Figure 9. Laser-stimulated fluorescence imaging (LSF; Kaye et al., 2015) of IVPP V22529 shows that the unknown structure has similar green fluorescence colours to dentine and maxillary bone. However, this does not help to constrain the identification of the unknown structure because cementum and ossified periodontal ligaments would probably fluoresce with similar colours since these materials are both made of fossilised hydroxylapatite. In the LSF image enamel reacts differently to the laser light even though it is also made of fossilised hydroxylapatite. Clearly, the mineralogy of the fossilised enamel is sufficiently different to the other parts of the fossil to give such a vividly different orange fluorescence colour.

Unfortunately, laser-stimulated fluorescence (LSF) analysis (using a 408nm violet laser; Kaye et al., 2015) was unable to support either the cementum 'jacket' or ossified ligament identifications. The LSF image (Fig. 9) shows similar greeny fluorescence colours for the unknown structure, dentine and maxillary bone, but a vivid orange colour for the enamel. This is interesting because all of these materials are varieties of fossilised hydroxylapatite (the ligaments are expected to have been ossified prior to fossilisation), but clearly there is a marked mineralogical difference between the fossilised enamel and the other fossilised materials that is probably related to mineral density. Thus, the unknown structures are proposed as cementum 'jackets' given the current evidence available.

To our knowledge these cementum 'jackets' have not been described amongst iguanodontians and other dinosaurs, but this derived root attachment tissue is commonly found in mammals where it also migrates onto the crowns (Erickson et al., 2012). Studies of existing iguanodontian specimens in person and from the literature revealed their presence in Equijubus normani (IVPP 12534, You et al., 2003c: Fig. 1E). Unlike IVPP V22529, these 'jackets' are found on alternating teeth rather than on each one. 


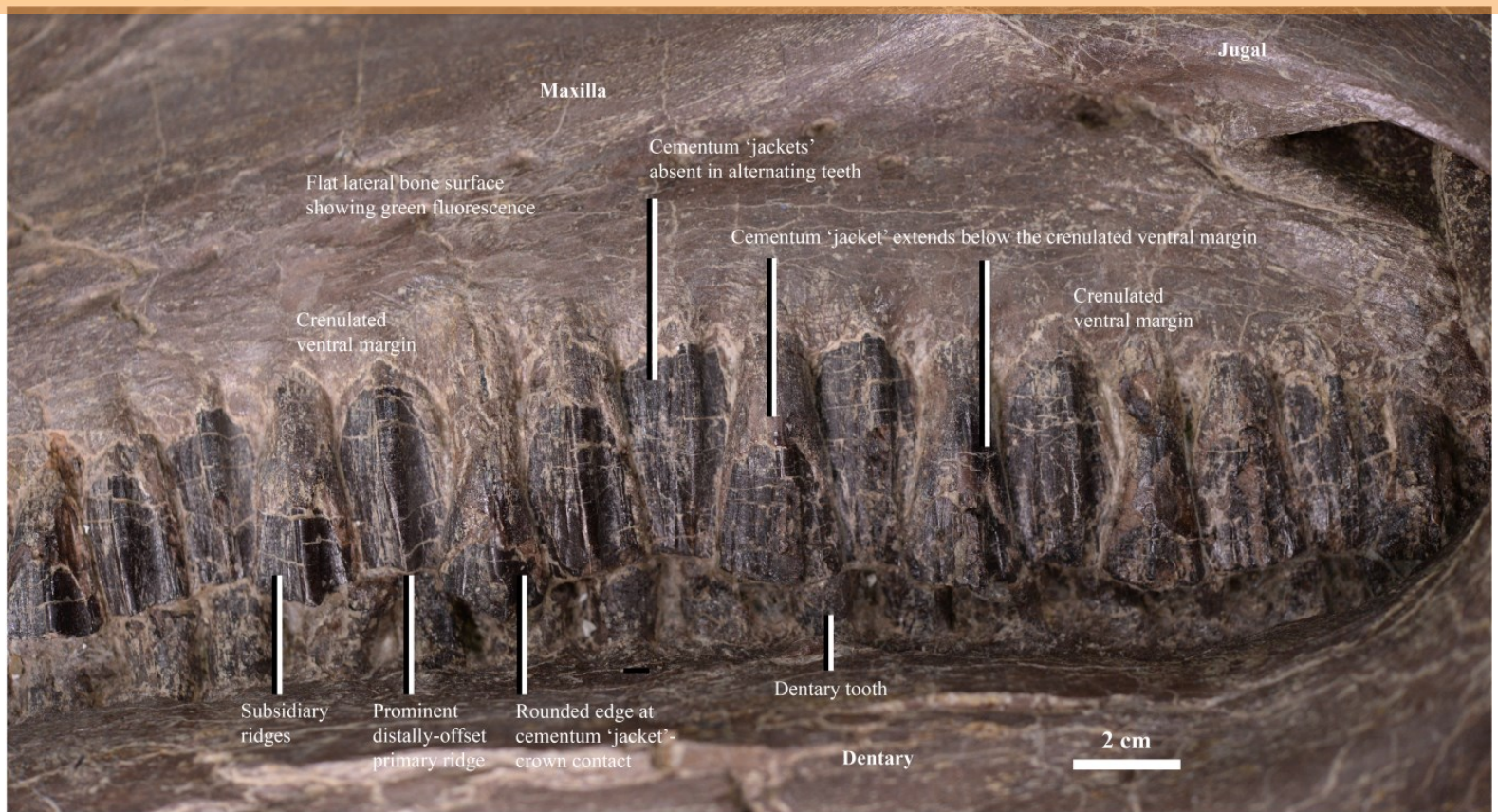

Figure 10. A cementum 'jacket' tooth morphology is also present in the basal hadrosauriform Equijubus normani (IVPP 12534, You et al., 2003c: Fig. 1E), but unlike IVPP V22529 this is observed in alternating teeth rather than on each tooth.

Tooth orientation

The maxillary tooth row follows a laterally concave path (Fig. 4). The slightly worn and unworn teeth appear to be posteriorly inclined whilst the heavily worn teeth are anteriorly inclined, but the latter appears to be an artefact of the fragmentation of the bone sockets holding them in place (Fig. 3). Thus, the specimen's tooth orientation is considered to be consistent with that of P. gobiensis (Norman, 2002) - posteriorly inclined.

\section{Maxillary body}

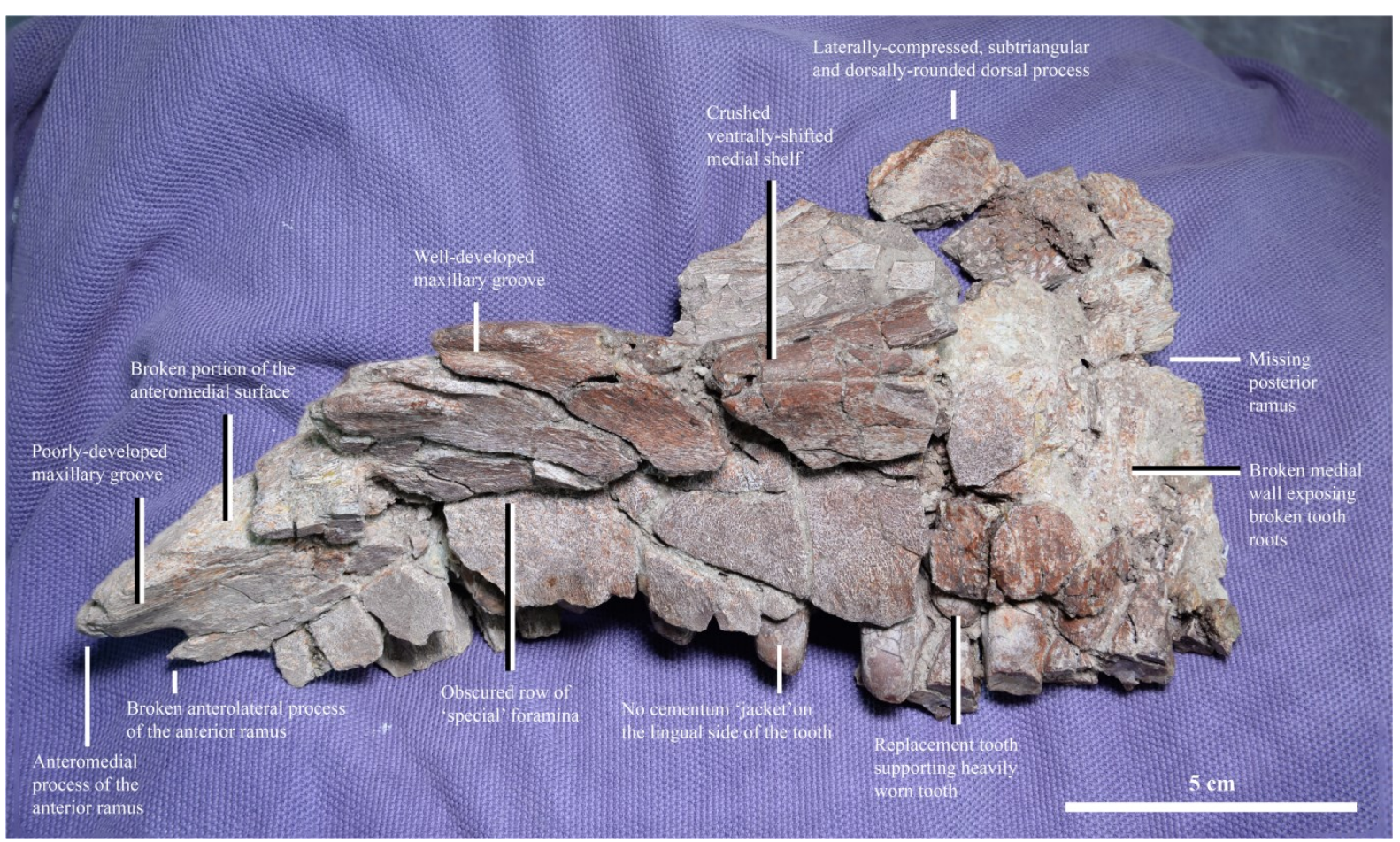


Figure 11. Maxilla in medial view showing the broken anterior ramus and missing posterior ramus as well as the maxillary grooves, medial shelf, dorsal process, lingual view of the tooth row and the broken posteromedial surface that exposes several fragmentary tooth roots.

The medial shelf (Fig. 11) has been artificially shifted ventrally partially obscuring the row of 'special' foramina such that their exact number and shapes are unclear; they are located relatively low on the medial surface, as in other basal hadrosauriforms such as Altirhinus (PIN 3386/7; right maxilla [Norman, 2002]). In Altirhinus (PIN 3386/7; Norman, 1998) an incomplete row of 14 regularly spaced foramina is preserved subparallel to the ventral margin of the maxilla (the missing posterior portion of the maxilla truncates this row of foramina). In Bactrosaurus their appearance varies between individuals of similar and different ages (adult/subadult: AMNH 6553, Fig. 7 Prieto-Márquez, 2011; juvenile: AMNH 6389, 6390, Figs. 9, 11 Prieto-Márquez, 2011). In Gilmoreosaurus there are at least 17 large, evenlyspaced and circular 'special' foramina (AMNH FARB 30653; Prieto-Márquez \& Norell, 2010).

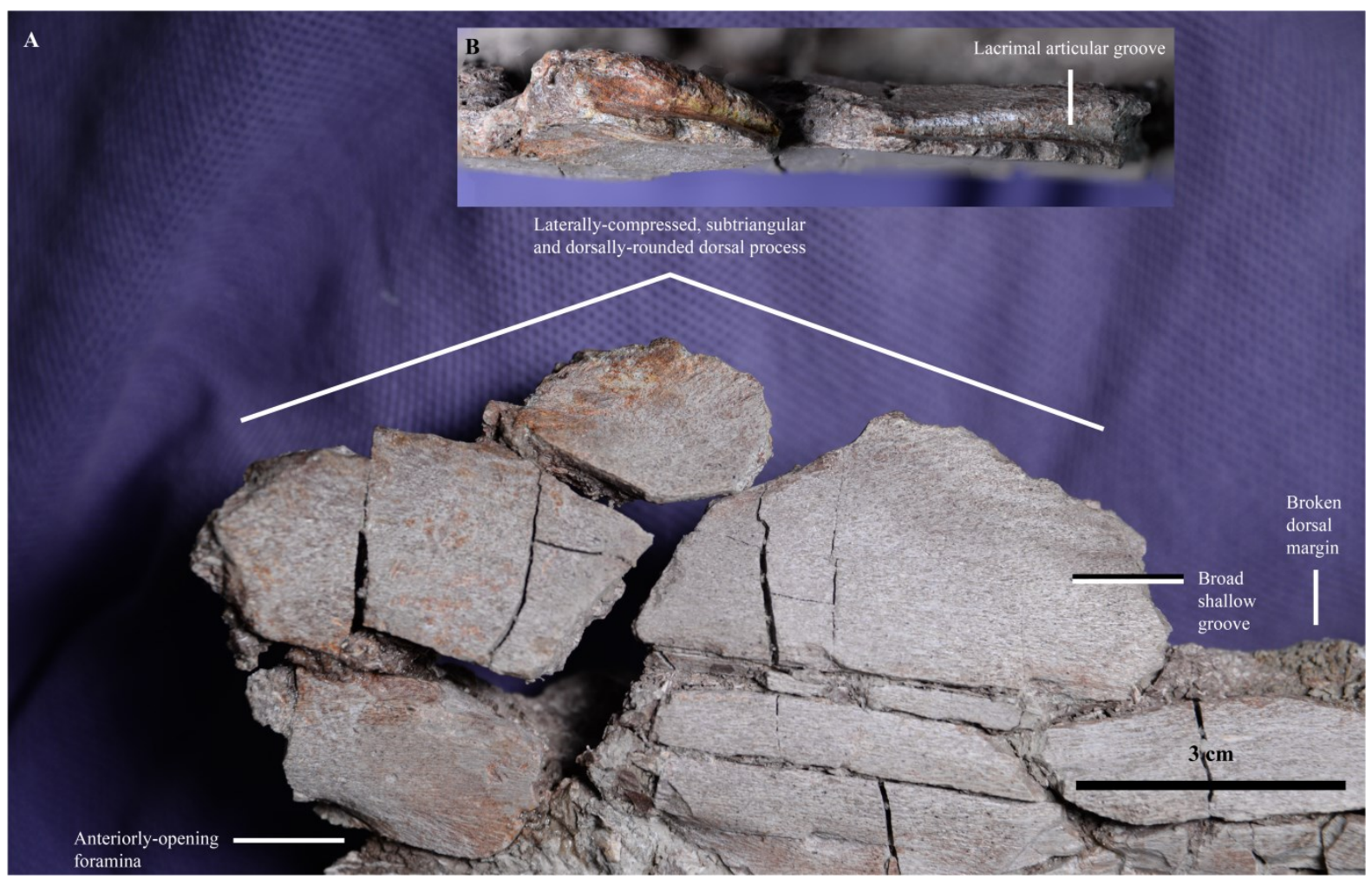

Figure 12. The dorsal process of IVPP V22529 is laterally-compressed, subtriangular and dorsally-rounded similar to the ones present in Altirhinus (PIN 3386/7; Norman, 2002: Fig. 6) and Bactrosaurus (AMNH 6389, 6390; Prieto-Márquez, 2011: Figs. 9, 11). Dorsal process in, A, lateral view; B, in dorsal view showing the lacrimal articular groove that is also present in Koshisaurus and Fukuisaurus [Shibata \& Azuma, 2015: Fig. 8C].

A relatively straight portion of the dorsal margin is preserved occupying around threequarters of the specimen's preserved anteroposterior length (Figs. 2, 11). This makes an $\sim 30^{\circ}$ angle with the ventral margin of the maxilla and is grooved (as observed in Koshisaurus and Fukuisaurus [Shibata \& Azuma, 2015: Fig. 8C]), presumably for articulation with the lacrimal (Fig. 12). The highest point of this section of preserved dorsal margin appears to preserve a laterally-compressed, subtriangular and dorsally-rounded dorsal (ascending) process similar to the ones present in Altirhinus (PIN 3386/7; Norman, 2002: Fig. 6) and 
Bactrosaurus (AMNH 6389, 6390; Prieto-Márquez, 2011: Figs. 9, 11). Norman (2015) characterises this process shape as a 'laterally flattened subtriangular plate' (Character 17, state 2) (Figs. 2, 11, 12). This process shape does not have a well-defined distribution amongst iguanodontians unlike the 'narrow' and 'finger-like' dorsal process morphologies that Norman (2015) recovered as synapomorphies of Iguanodontia and Ankylopollexia respectively (state 0 of character 17 respectively under ACCTRAN [='narrow, figure-like process']). The dorsal process of IVPP V22529 possesses a shallow subcircular depression on its lateral surface beneath its tip (Figs. 2, 11, 12) which is not observed in Bactrosaurus and Altirhinus. A comparison with the condition in Probactrosaurus is not possible as the dorsal region is not preserved e.g. in PIN 2232/9-2 and /10-2 (Norman, 2002) [coded as a '?' in Norman, 2015]. IVPP V22529 is not sufficiently well-preserved to confidently characterise the presence or absence of an antorbital fenestra, a feature that is used to diagnose clypeodont ornithischians and their subclades (Norman, 2015).

The anterior portion of the maxilla appears to be subtriangular (Figs. 2, 11, 13), as in most iguanodontians including Altirhinus (PIN 3386/7; Norman, 1998: Fig. 6B), Bactrosaurus (AMNH 6553; Prieto-Márquez, 2011) and Probactrosaurus (PIN 2232/9-2; Norman, 2002). The anterior maxillary ramus of IVPP V22529 is forked into a pointed anteromedial process at around the same level as the incompletely preserved anterolateral process (Figs. 2, 11, 13). Owing to the incomplete preservation of the latter process (Figs. 2, 11, 13), the relative length and size of these processes cannot be determined. Processes of different sizes are found in the 'iguanodontoid' styracosternan Iguandon (Weishamphel et al., 1993), Protohadros (Head, 1998: Fig. 3C, D), Bactrosaurus (AMNH 6553, 6389, 6390; Prieto-Márquez, 2011: Figs. 712) and Koshisaurus (Shibata \& Azuma, 2015: Figs. 3A, C; 8A, B, E), but in the latter the anterior processes are actually of similar rather than different lengths (Shibata \& Azuma, 2015: Fig. 3A). Bifurcated anterior processes are actually diagnostic of Iguanodontia (Norman, 2015: character 15, state 1; ACCTRAN), but in the left maxilla of Shuangmiaosaurus the anterolateral process appears to have become particularly enlarged with a dorsally placed nubbin at its base potentially being the remnants of the anteromedial process (LPM0165; You et al., 2003b: Fig. 1A). Teeth are present right up to base of the anteromedial process, as in Protohadros (Head, 1998: Fig. 3C, D). The anterior half of the anterodorsal margin of the anterolateral process of IVPP V22529 has a finger-shaped recess (rostral foramen) (Fig. 13), as in non-hadrosaurid iguandontians. 


\section{Peer Preprints n NOTPEER-REVIEWED}

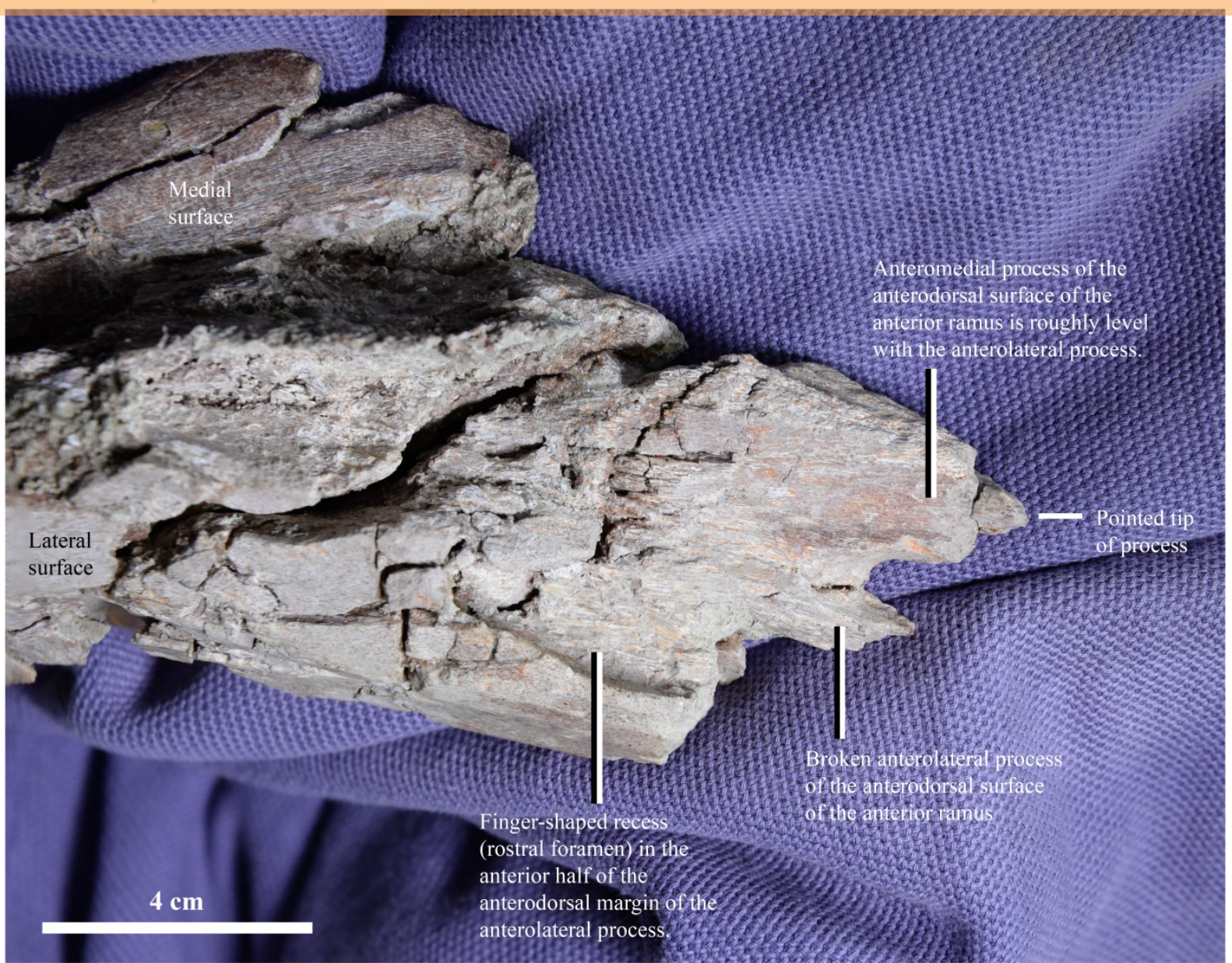

Figure 13. Dorsal view of the anterodorsal process of IVPP V22529 showing the bifurcating anterior processes at roughly the same level. Only the pointed anteromedial process is complete. This is a finger-shaped recess (rostral foramen) in the anterior half of the anterodorsal margin of the anterolateral process.

Halfway up the medial side of the maxilla there is a well-developed medially projecting shelf that originates from the dorsomedial portion of the anterior ramus (Fig. 11). Subhorizontal ridges along the medial side of the anteromedial process and the medial shelf become increasingly well-developed dorsoposteriorly (although a portion of the intervening area is broken). These ridges demark the boundaries of the maxillary grooves. Unlike IVPP V22529, the maxillary grooves of Koshisaurus (Shibata \& Azuma, 2015: Figs. 3C; 8B, E) are all poorly-developed where there are all well-developed in Protohadros (SMU 74582; Head, 1998: Fig. 3D) and Fukuisaurus (FPDM-V40-1; Shibata \& Azuma, 2015: Fig. 8B, E; three and five grooves respectively). Maxillary grooves presumably relate to the attachment of soft tissues in the roof of the mouth, but these seems to be absent in the most derived iguanodontians - the distribution of this feature is unclear across Iguanodontia. The evolution of maxillary grooves is therefore of interest in further understanding iguanodontian feeding and as a potential source of phylogenetic information. 
419

420

421

422

423

424

425

426

427

428

429

430

431

432

433

434

435

436

437

438

439

440

441

442

443

444

445

446

447

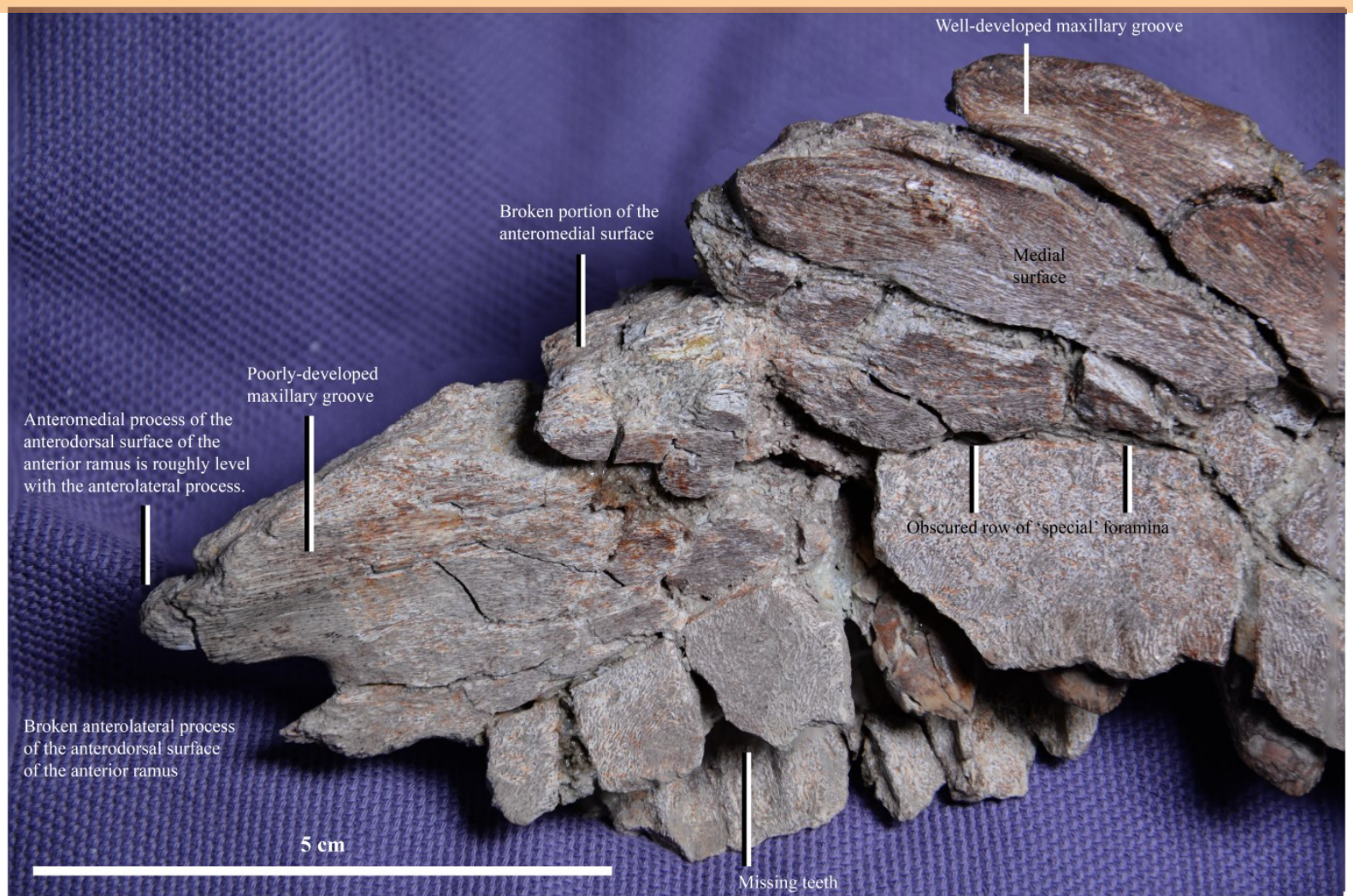

Figure 14. The maxillary grooves in IVPP V22529 become better-developed along the medial surface of the anteromedial process and the medial shelf, unlike in Protohadros (SMU 74582; Head, 1998: Fig. 3D), Fukuisaurus (FPDM-V40-1; Shibata \& Azuma, 2015: Fig. 8B, E) and Koshisaurus (Shibata \& Azuma, 2015: Figs. 3C; 8B, E) where the grooves in each specimen are of similar sizes. However, the phylogenetic significance of maxillary groove morphologies is not understood.

The mediolateral width of the medial shelf is affected by dorsoventral diagenetic compression, as evident from bone fragments that are thrusted upon each other (Fig. 11). However, the exact extent of this diagenetic artefact is unclear because a compressioncorrected bone reconstruction is beyond the scope of this paper to produce. Bactrosaurus has a comparatively less developed shelf in both adult (AMNH 6553; Prieto-Márquez, 2011: Fig. 7) and juvenile specimens (AMNH 6389, 6390; Prieto-Márquez, 2011: Figs. 9, 11) as well as Altirhinus (PIN 3386/7; Norman, 1998: Fig. 6B) [but the degree of lesser development is unknown for the aforementioned reason]. In these specimens and in IVPP V22529 the shelf is angled slightly dorsoposteriorly (Fig. 11). In contrast, Probactrosaurus was described by Norman (2002) as having a vertical and planar medial wall.

The entire posterior portion of IVPP V22529 is missing so the morphology of the jugalmaxilla suture is unknown (Figs. 2, 11). In Altirhinus (PIN 3386/7; Norman, 1998: Fig. 6A) the jugal sutural surface is a finger-like process that fits into a slot in the anterior ramus of the jugal, a feature that unites styracosternan iguanodontians (Norman, 2015: character 20, state 1). The missing anterodorsal margin makes it impossible to infer the morphology of the jugal's ventral margin which is sinusoidal in ankylopollexian iguanodontians (Norman, 2015: character 21, state 1). The missing posterior ramus means it is unclear if this is dorsoventrally tall with a rounded but slightly irregular tip, as in Probactrosaurus (PIN 2232/9-2,10-2; Norman, 2002: Fig. 5), or if it is more 'finger-like', as in Altirhinus (PIN 3386/7; Norman, 2002, Fig. 6A, B). 


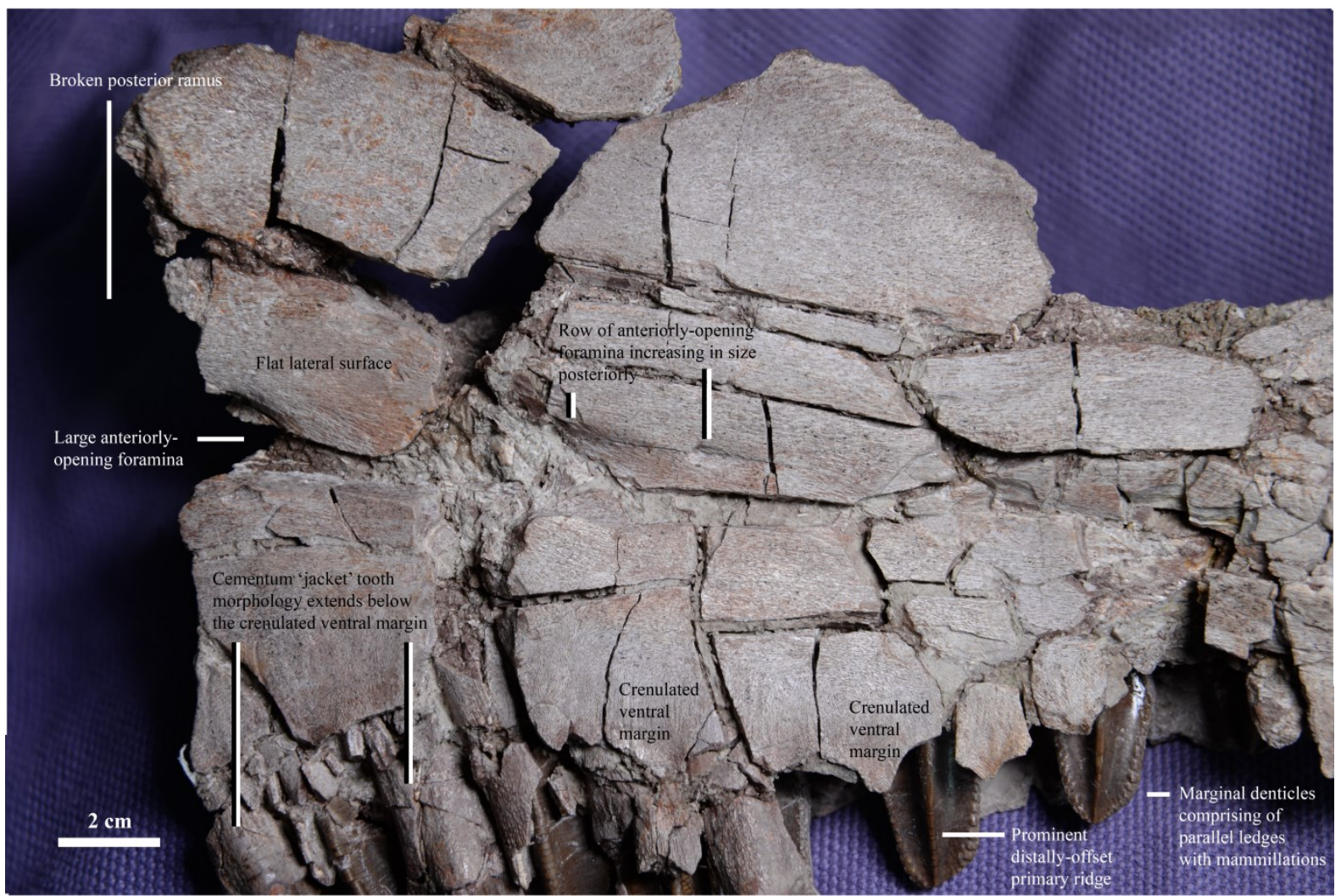

449

Figure 15. Close-up of the relatively flat lateral surface of IVPP V25529 showing a row of anteriorly-opening foramina that increase in size posteriorly.

The lateral surface of the maxilla is relatively flat, a trait that is undoubtedly influenced by the fragmentation of the specimen (Figs. 2, 15). Ventral to the dorsal process is a broad shallow groove (Figs. 2, 12). There is a low scattered row of five anteriorly-opening neurovascular foramina on the lateral surface that increase in size posteriorly (Fig. 15). These observations appear to be generally consistent with Probactrosaurus, notably the right maxilla of PIN 2232-10-2 (Norman, 2002, Fig. 5A), except that the foramina in the latter do not increase in size posteriorly. However, these arguments are weakened by the absence of foramina in the larger left maxilla Probactrosaurus PIN 2232-9-2 (Norman, 2002, Fig. 5B) suggesting that these foramina are not consistently expressed on the sides of the skull and/or they may undergo changes with age or be different between sexes.

\section{Discussion}

Taxonomic status of IVPP V22529

\section{A basal hadrosauriform based on tooth anatomy}

IVPP V22529 matches the maxillary teeth characteristics in the diagnosis of Probactrosaurus (Norman, 2002): 'maxillary teeth narrow with prominent primary ridge and no subsidiary ridges; tall and interlocking teeth that form a high, posteriorly inclined battery; marginal denticles are mammillate.' However, these characteristics are not used to refer IVPP V22529 to Probactrosaurus (Norman, 2002) as the aforementioned characteristics are now understood to have a wider distribution amongst iguanodontians than previously appreciated (Norman, 2015). The tooth anatomy of IVPP V22529 identifies it as a non-euhadrosaurian 
hadrosauriform: two or more replacement crowns are a hadrosauriform iguandontian feature (Norman, 2015: character 54, state 1) whilst the absence of a single median primary ridge is a non-euhadrosaurian iguanodontian feature (Norman, 2015: character 68, state 3). The marginal denticles of IVPP V22529 comprise of parallel ledges with single rows of $\sim 6$ relatively large mammillae, a feature that further constrains the specimen as a basal hadrosauriform.

\section{IVPP V22529 compared to Probactrosaurus}

IVPP V22529 has a number of noteworthy differences with Probactrosaurus maxillae, despite the former missing its posterior portion and the latter missing anterior ramii. Firstly, Norman (2002) noted that Probactrosaurus gobiensis has a vertical and planar medial wall whereas in IVPP V22529 this in non-planar owing to its well-developed medial shelf (Fig. 11). However, a small proportion of the latter is ascribed to specimen deformation. Secondly, there is a low scattered row of five anteriorly-opening foramina on the lateral surface of IVPP V22529 that increase in size posteriorly, but in Probactrosaurus PIN 2232-10-2 (Norman, 2002, Fig. 5A) these foramina do not increase in size posteriorly and are even absent in specimen PIN 2232-9-2 (Norman, 2002, Fig. 5B). In Probactrosaurus (and iguanodontians more generally) there is incomplete knowledge of how maxillary foramina change with age and how they can be different between sexes or individuals. Thus, further work is needed to evaluate these types of variability so that the phylogenetic utility of maxillary foramina can be established. Therefore, erring on the side of caution, the foraminal differences between IVPP V22529 and Probactrosaurus should be considered tentatively as differences with taxonomic value, and certainly warrant lesser value than the aforementioned medial shelf difference. The presence and absence of the unique cementum 'jackets' in IVPP V22529 and Probactrosaurus respectively could be strong evidence for differentiating them. However, given the areas of uncertainty in the identification and formation mechanism of this structure as well as its presence in at least one iguanodontian, it would be inappropriate to place phylogenetic value on this structure until it is more extensively investigated. The teeth of IVPP V22529 and Probactrosaurus are very similar and their maxillae are both subtriangular.

\section{IVPP V22529 compared to Bactrosaurus and Gilmoreosaurus}

The expression of neurovasucular foramina on the lateral surface of the maxilla is variably expressed in Bactrosaurus, which has a row of different-sized ones in juvenile specimen AMNH 6389 (Prieto-Márquez, 2011: Fig. 9) but more random arranged one in juvenile specimen AMNH 6390 (Prieto-Márquez, 2011: Fig. 11). However, as in IVPP V22529 these foramina appear low on the lateral surface. In contrast, the maxillary foramina of Gilmoreosaurus AMNH FARB 30653 (Prieto-Márquez \& Norell, 2010) are more randomly distributed but appear high as well as low on the lateral surface. On the medial surface of the latter specimen the 'special foramina' are larger and more circular than those in IVPP V22529 (although these are partially obscured by the displaced medial shelf) and Bactrosaurus (AMNH 6553, 6389, 6390; Prieto-Márquez, 2011: Figs. 8, 10, 12). Gilmoreosaurus AMNH FARB 30653 (Prieto-Márquez \& Norell, 2010) and Bactrosaurus (AMNH 6553, 6389, 6390; Prieto-Márquez, 2011: Figs. 8, 10, 12) lack maxillary grooves that become increasingly developed along the medial surface of the anteromedial process and the medial shelf, as in IVPP V22529 (Fig. 11). However, Gilmoreosaurus AMNH FARB 30653 (Prieto-Márquez \& Norell, 2010) has less-developed maxillary grooves restricted to the medial surface of the anteromedial process and so are less extensive than those of Koshisaurus (Shibata \& Azuma, 2015: Figs. 3C; 8B, E). Bactrosaurus AMNH 6389 (Prieto- 
Márquez, 2011: Fig. 10) also appears to share this characteristic with Gilmoreosaurus, but this needs to be confirmed by first-hand study of this specimen.

Cementum 'jackets'

Cementum 'jackets' are a dental structure presumably related to feeding style, but how it is related to this is not yet obvious. The restriction of these 'jackets' to the labial side of the tooth appears to be genuine and suggests this side was well-anchored to the tooth socket. The latter trait may have been important in allowing the 'jackets' to help resist lateral components of bite forces, a force regime that is evident from the medially-directed slope of the wear facets of the teeth. The 'jackets' probably reduced stress on the brittle tooth crests more generally as well by transmitting loads amongst the tissues of the teeth (Erickson et al., 2012). However, if these structures did have these roles then the alternating occurrence of the 'jackets' in Equijubus implies that not all taxa benefitted from this hypothesised function equally. Histological analysis will no doubt be invaluable in testing the cementum 'jacket' hypothesis at the microscopic level and should help to clarify both the composition and morphology of these structures. Unfortunately, such work is beyond the scope of the current study, but it should be a priority for future studies of IVPP V22529. Future opportunities to compare feeding biomechanics in IVPP V22529 (and other basal hadrosauriformes) with more derived hadrosauriforms (Erickson et al., 2012) will also be worthwhile to determine how important these cementum 'jackets' were in iguandontian dental system evolution.

\section{Conclusions}

IVPP V22529 is an isolated Early Cretaceous partial right iguanodontian maxilla that possesses tooth characteristics identifying it as a basal hadrosauriform. However, other parts of this bone fail to convincingly support a referral to a new or existing taxon, including to a new or existing species of Probactrosaurus, a contemporaneous genus known from the same locality in North China. Further work may better constrain the taxonomic status of this specimen if characteristics differing from Probactrosaurus can be validated, namely: a unique corrugated middle ventrolateral margin, a row of foramina on its lateral surface that open anteriorly and increasing in size posteriorly as well as a prominent medial shelf. Despite its coarse level of identification, IVPP V22529 has important implications for our understanding of iguanodontian (and dinosaurian) dental architecture. In labial view, five middle maxillary crowns each preserve a rugose cementum sheath over their basal portions that are separate from the tooth socket but actually originate within them and extend ventrally below the crenulated ventral margin of the maxilla. This arrangement forms a corrugated ventrolateral surface as grooves separate the sides of these sheaths. This structure - which we propose to call a cementum 'jacket' structure - appears to be present in the basal hadrosauriform Equijubus as well, but this differs from IVPP V22529 in being present in every other tooth rather than on each tooth. To our knowledge this structure has not been described in other dinosaurs, but cementum commonly migrates onto the tooth crowns of mammals (Erickson et al., 2012). The wider distribution of cementum 'jackets' amongst iguanodontians (and dinosaurs more generally) warrants further attention as their morphology could carry important phylogenetic information. The restriction of these 'jackets' to the labial face of the teeth might indicate a structural role in resisting the lateral component of bite forces and/or the stress on the brittle tooth crests, but these hypotheses and confirmation of 'jacket' composition and morphology would greatly benefit from future histological analysis and biomechanical studies that were beyond the scope of this study. Despite, the further work required, IVPP V22529 provides important new insights into the dental architecture of basal 
hadrosauriforms that deepens our understanding of the morphological diversity that preceded the revolutionary advanced hadrosauriform dental battery system (Erickson et al., 2012).

\section{Financial statement}

The fieldwork was supported by the National Science Foundation of China (41128002; 41120124002) and the Hundred Talents Programs of the Chinese Academy of Sciences. Study of IVPP V22529 was supported by the University of Hong Kong's Faculty of Science.

\section{Acknowledgements}

We would like to thank Yu Tao, Li Shuo and Marvin Meng who were also part of the 2015 IUP-AMNH-HKU Maortu field expedition. IVPP V22529 was prepared by Ding Xiaoqin and was discovered by Jin Meng. Zhang Hailong is thanked for taking the photograph in Figure 5. Xing Hai is also thanked for his comments and discussion during this study which helped improve the quality of this manuscript.

\section{References}

Barrett PM, Butler RJ, Wang XL, and Xu X. 2009. Cranial anatomy of the iguanodontoid ornithopod Jinzhousaurus yangi from the Lower Cretaceous Yixian Formation of China. Acta Palaeontologica Polonica 54:35-48.

Brusatte SL, Benson RBJ, Chure DJ, Xu X, Sullivan C, and Hone DWE. 2009. The first definitive carcharodontosaurid (Dinosauria: Theropoda) from Asia and the delayed ascent of tyrannosaurids. Naturwissenschaften 96:1051-1058.

Brusatte SL, Chure DJ, Benson RBJ, and Xu X. 2010. The osteology of Shaochilong maortuensis, a carcharodontosaurid (Dinosauria: Theropoda) from the Late Cretaceous of Asia. Zootaxa 2334:1-46.

Buffetaut E, and Suteethorn V. 2011. A new iguanodontian dinosaur from the Khok Kruat Formation (Early Cretaceous, Aptian) of northeastern Thailand. Annales de Paléontologie 97:51-62.

Chow M, and Rozhdestvensky AK. 1960. Exploration of Inner Mongolia: a preliminary account of the 1959 field work of the Sino-Soviet paleontological expedition (SSPE). Vertebrata Palasiatica 4:1-10.

Erickson GM, Krick BA, Hamilton M, Bourne GR, Norell MA, Lilleodden E, and Sawyer WG. 2012. Complex dental structure and wear biomechanics in hadrosaurid dinosaurs. Science 338:98-101.

Godefroit P, Dong ZM, Bultynck P, Li H, and Feng L. 1998. Sino-Belgian Cooperative Program - Cretaceous dinosaurs and mammals from Inner Mongolia: 1) New Bactrosaurus (Dinosauria: Hadrosauroidea) material from Iren Dabasu (Inner Mongolia, P.R. China). Bulletin de l'Institute Royal des Sciences Naturelles du Belgique 68:1-70.

Godefroit P, Escuillié F, Bolotsky YL, and Lauters P. 2012. A new basal hadrosauroid dinosaur from the Upper Cretaceous of Kazakhstan. In: Godefroit P, ed. Bernissart dinosaurs and Early Cretaceous terrestrial ecosystems. Bloomington: Indiana University Press, 334-358.

Hans-Volker K. 1999. Paleogeography and systematics of the genus Dogania Gray, 1844 (Testudines: Trionychidae). Studia Geological Salmanticensia 35:3-8. 
Head JJ. 1998. A new species of basal hadrosaurid (Dinosauria, Ornithopoda) from the Cenomanian of Texas. Journal of Vertebrate Paleontology 18:718-738.

Horner JR, Weishampel DB, and Forster CA. 2004. Hadrosauridae. In: Weishampel DB, Dodson P, and Osmólska H, eds. The Dinosauria. Berkeley: University of California Press, 438-463.

Hübner TR, and Rauhut OWM. 2010. A juvenile skull of Dysalotosaurus lettowvorbecki (Ornithischia: Iguanodontia), and implications for cranial ontogeny, phylogeny, and taxonomy in ornithopod dinosaurs. Zoological joumal of the Linnean Society 160:366-396.

Kaye TG, Falk AR, Pittman M, Sereno PC, Martin LD, Burnham DA, Gong EP, Xu X, and Wang Y. 2015. Laser-stimulated fluorescence in paleontology. PLoS One 10:e0125923.

Kirkland JI. 1998. A new hadrosaurid from the upper Cedar Mountain Formation (AlbianCenomanian: Cretaceous) of eastern Utah - the oldest known hadrosaurid (lambeosaurine?). In: Lucas SG, Kirkland JI, and Estep JW, eds. Lower and Middle Cretaceous terrestrial ecosystems: New Mexico Museum of Natural History and Science Bulletin, 283-295.

Kobayashi Y, and Azuma Y. 2003. A new iguanodontian (Dinosauria; Ornithopoda), form the lower Cretaceous Kitadani Formation of Fukui Prefecture, Japan. Journal of Vertebrate Paleontology 23:166-175.

Lü JC. 1997. A new Iguanodontidae (Probactrosaurus mazongshanensis sp. nov.) from Mazongshan Area, Gansu Province, China. In: Dong Z, ed. Sino-Japanese Silk Road Dinosaur Expedition. Beijing: China Ocean Press, 27-47.

Norman DB. 1998. On Asian ornithopods (Dinosauria: Ornithischia). 3. A new species of iguanodontid dinosaur. Zoological joumal of the Linnean Society 122:291-348.

Norman DB. 2002. On Asian ornithopods (Dinosauria: Ornithischia). 4. Probactrosaurus Rozhdestvensky, 1966. Zoological Journal of the Linnean Society 136:113-144.

Norman DB. 2015. On the history, osteology, and systematic position of the Wealden (Hastings group) dinosaur Hypselospinus fittoni (Iguanodontia: Styracosterna). Zoological Journal of the Linnean Society 173:92-189.

Norman DB, Hilpert KH, and Hölder H. 1987. Die Wirbeltierfauna von Nehden (Sauerland), Westdeutschland. Geologie und Paläontologie in Westfalen 8:1-77.

Prieto-Márquez A. 2011. Cranial and appendicular ontogeny of Bactrosaurus johnsoni, a hadrosauroid dinosaur from the Late Cretaceous of Northern China. Palaeontology 54:773-792.

Prieto-Márquez A, and Norell MA. 2010. Anatomy and relationships of Gilmoreosaurus mongoliensis (Dinosauria: Hadrosauroidea) from the Late Cretaceous of Central Asia. American Museum Novitates 3694:1-49.

Rozhdestvensky AK. 1966. Novyye iguanodonty iz Tsentral'noy Azii. Filogeneticheskiye i taksonomicheskiye $\mathrm{v}$ zaimootnosheniya pozdnikh Iguanodontidae i rannikh Hadrosauridae [New iguanodonts from Central Asia. Phylogenetic and taxonomic interrelationships of late Iguanodontidae and early Hadrosauridae]. Palaeontologicheskii Zhurnal 1966:103-116.

Rozhdestvensky AK. 1974. Istoria dinosavrovikh faun Asii i drugich materikov i vopros paleogeografii [Dinosaur faunal history in Asia and its bearing on palaeogeography]. Fauna I Biostratigrafia Mesozoiya I Kainozoiy Mongolii. Moskva: Akademiya Nauk, 107-131.

Shibata M, and Azuma Y. 2015. New basal hadrosauroid (Dinosauria: Ornithopoda) from the Lower Cretaceous Kitadani Formation, Fukui, central Japan. Zootaxa 3914:421-440. 
van Itterbeeck J, Bultynk P, Li GW, and Vandenburghe N. 2001. Stratigraphy, sedimentology and palaeoecology of the dinosaur-bearing Cretaceous strata of Dashuiguo (Inner Mongolia, People's Republic of China). Bulletin de l'Institut Royal des Sciences Naturelles de Belgique 71:51-70.

van Itterbeeck J, Markevich JS, and Horne DJ. 2004. The age of the dinosaur-bearing Cretaceous sediments at Dashuiguo, Inner Mongolia, P.R. China based on charophytes, ostracods and palynomorphs. Cretaceous Research 25:391-409.

Vickaryous MK, Russell AP, Currie PJ, and Zhao XJ. 2001. A new ankylosaurid (Dinosauria : Ankylosauria) from the Lower Cretaceous of China, with comments on ankylosaurian relationships. Canadian Journal of Earth Sciences 38:1767-1780.

Vitek NS, and Danilov IG. 2010. New material and a reassessment of soft-shelled turtles (Trionychidae) from the Late Cretaceous of Middle Asia and Kazakhstan. Journal of Vertebrate Paleontology 30:383-393.

Wang XL, and Xu X. 2001. A new iguanodontid (Jinzhousaurus yangi gen. et sp. nov.) from the Yixian Formation of western Liaoning, China. Chinese Science Bulletin 46:16691672.

Weishamphel DB, Grigorescu D, and Norman DB. 1993. Telmatosaurus transsylvanicus from the Late Cretaceous of Romania: the most basal hadrosaurid dinosaur. Palaeontology 36:361-385.

$\mathrm{Wu}$ WH, and Godefroit P. 2012. Anatomy and relationships of Bolong yixianensis, an Early Cretaceous iguanodontoid dinosaur from western Liaoning, China. In: Godefroit P, ed. Bernissart dinosaurs and Early Cretaceous terrestrial ecosystems. Bloomington: Indiana University Press, 174-212.

Wu WH, Godefroit P, and Hu DY. 2010. Bolong yixianensis gen. et sp. nov.: A new iguanodontoid dinosaur from the Yixian Formation of Western Liaoning, China. Geology and Resources 19:127-133.

Yeh HK. 1965. New materials of fossil turtles of Inner Mongolia. Vertebrata Palasiatica 9:47-69.

You HL, Ji Q, and Li DQ. 2005. Lanzhousaurus magnidens gen. et sp. nov. from Gansu Province, China: the largest-toothed herbivorous dinosaur in the world. Geological Bulletin of China 24:785-794.

You HL, Ji Q, Li JL, and Li YX. 2003a. A new hadrosauroid dinosaur from the MidCretaceous of Liaoning, China. Acta Geologica Sinica - English Edition 77:148-154.

You HL, Ji Q, Li JL, and Li YX. 2003b. A new hadrosauroid dinosaur from the midCretaceous of Liaoning, China. Acta Geological Sinica 77:148-155.

You HL, Li DQ, and Liu WC. 2011. A new hadrosauriform dinosaur from the Early Cretaceous of Gansu Province, China. Acta Geologica Sinica 85:51-57.

You HL, Luo ZX, Shubin NH, Witmer LM, Tang XL, and Tang F. 2003c. The earliestknown duck-billed dinosaur from deposits of late Early Cretaceous age in northwest China and hadrosaur evolution. Cretaceous Research 24:347-355.

Zheng WJ, Jin XS, Shibata M, and Azuma Y. 2013. An early juvenile specimen of Bolong yixianensis (Ornithopoda: Iguanodontia) from the Lower Cretaceous of Ningcheng County, Nei Mongol, China. Historical Biology 26:236-251. 